\title{
The effect of parameters on positive
} solutions and asymptotic behavior of an unstirred chemostat model with B-D functional response

\author{
Xiaozhou Feng ${ }^{1,2^{*}}$, Suping Sun ${ }^{1}$, Tongqian Zhang ${ }^{3,4}$ and Xiaomin $\mathrm{An}^{1}$
}

"Correspondence:

flxzfxz8@163.com

'Department of Mathematics and

Physics, Xi'an Technological

University, Xi'an, P.R. China

${ }^{2}$ College of Mathematics, Shaanxi

Normal University, Xi'an, P.R. China

Full list of author information is

available at the end of the article

\section{第 Springer}

\begin{abstract}
This paper deals with the effect of parameters on properties of positive solutions and asymptotic behavior of an unstirred chemostat model with the Beddington-DeAngelis (denote by B-D) functional response under the Robin boundary condition. Firstly, we establish some a priori estimates and a sufficient condition for the existence of positive solutions (see (Feng et al. in J. Inequal. Appl. 2016(1):294, 2016)). Secondly, we study the effect of the small parameter $k_{1}$ and sufficiently large $k_{2}$ in B-D functional response, which shows that the model has at least two positive solutions. Thirdly, we investigate the case of sufficiently large $k_{1}$. The results show that if $k_{1}$ is sufficiently large, then the positive solution of this model is determined by a limiting equation. Finally, we present an asymptotic behavior of solutions depending on time. The main methods used in this paper include the fixed point index theory, bifurcation theory, perturbation technique, comparison principle, and persistence theorem.
\end{abstract}

MSC: $35 J 57 ; 35 K 51 ; 35 K 57$

Keywords: Chemostat; Positive solutions; The fixed point index theory; Multiplicity

\section{Introduction}

The chemostat is a very important resource-based model for the continuous culture of competition microorganisms and a standard model for the laboratory apparatus on bioreactor, which have been studied from various views such as population dynamics and species interactions [1-17]. For early works, we refer to [2-4]. The chemostat model of competition for a single-limit nutrient between plasmid-bearing and plasmid-free organisms was proposed by Stephanopoulos and Lapidus [13], who established some local results, whereas a global result was presented in [14]. The inhibition effects on plasmid populations were studied by Hsu and Waltman [15]. The chemostat model with impulsive input nutrient concentration was studied from different views in [18-22]. The above research of the chemostat model is related to the ODE model. Recently, the coexistence and stability of chemostat models were studied from the viewpoint of PDE (see Wu [5-7], Nie $[8,9,16]$, Wang [17], and Zhang [23]), which can better simulate the unstirred chemostat model.

(c) The Author(s) 2018. This article is distributed under the terms of the Creative Commons Attribution 4.0 International License (http://creativecommons.org/licenses/by/4.0/), which permits unrestricted use, distribution, and reproduction in any medium, provided you give appropriate credit to the original author(s) and the source, provide a link to the Creative Commons license, and indicate if changes were made. 
In this paper, we are concerned with the following unstirred chemostat model with the B-D functional response under homogeneous Robin boundary condition in a bounded domain $\Omega$ :

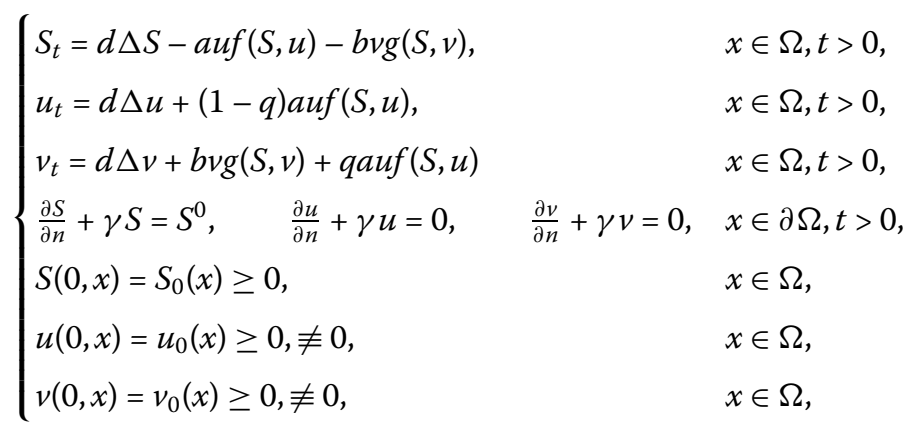

where $R^{N}(N \geq 1)$ is a bounded domain with smooth boundary $\partial \Omega, a, b, m_{i}, k_{i}(i=1,2)$, and $r$ are positive constants, the biological background of which is described in [1], $S$ stands for the densities of the nutrient, $u$ and $v$ are the densities of the plasmid-bearing and plasmidfree organisms, respectively, and the parameter $q(0<q<1)$ stands for the fraction of plasmid-bearing organism converting into plasmid-free organism. Here $f(S, u)=\frac{S}{1+m_{1} S+k_{1} u}$ and $g(S, v)=\frac{S}{1+m_{2} S+k_{2} v}$ are the Beddington-DeAngelis (B-D) functions (see [11, 24]), supported by numerous laboratory experiments and observations and providing better description of predator feeding over a range of predator-prey abundances.

Note that Nie and Wu [16] studied the coexistence of an unstirred chemostat model with Beddington-DeAngelis functional response and inhibitor, but the parameters of B-D functional response is different from (1), and two models are essentially different. Meanwhile, Wang et al. [17] also obtained the coexistence and stability of an unstirred chemostat model with the Beddington-DeAngelis function, but their model does not include the plasmid transformation of two competition species. However, this paper deals with plasmid-bearing and plasmid-free models in the unstirred chemostat with the B-D functional response under a homogeneous Robin boundary condition.

In [1], the coexistence of an unstirred chemostat model (1) with B-D functional response is established by fixed point index theory, but in the present paper, we investigate the effect of parameters on the multiplicity and stability of positive solutions of equilibrium state model of (1); moreover, the asymptotic behavior of solutions of (1) is established, which further enrich the results for system (1). Now, we are concerned with the following elliptic system:

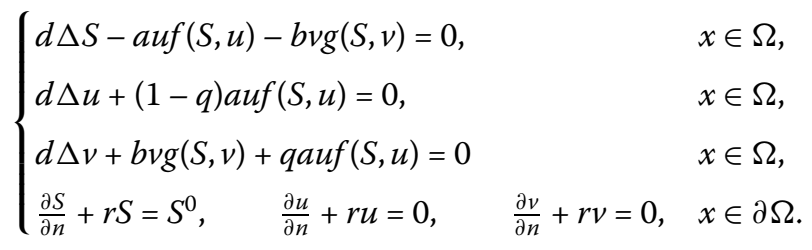

Let $z=S+u+v$. Then $S=z-u-v$ and $z$ satisfy

$$
\Delta z=0, \quad x \in \Omega, \quad \frac{\partial z}{\partial v}+r z=S^{0}, \quad x \in \partial \Omega
$$


By [1] we directly obtain the equivalent system of (2) as follows:

$$
\begin{cases}d \Delta u+(1-q) a u f(z-u-v, u)=0, & x \in \Omega, \\ d \Delta v+b v g(z-u-v, v)+q a u f(z-u-v, u)=0, & x \in \Omega, \\ \frac{\partial u}{\partial n}+r u=0, \quad \frac{\partial v}{\partial n}+r v=0, & x \in \partial \Omega,\end{cases}
$$

where $f(z-u-v, u)=\frac{z-u-v}{1+m_{1}(z-u-v)+k_{1} u}$ and $g(z-u-v, v)=\frac{z-u-v}{1+m_{2}(z-u-v)+k_{2} v}$. By a method similar to [11], positive solutions of (3) must meet

$$
S(x)+u(x)+v(x)=z(x), \quad x \in \bar{\Omega} .
$$

The rest of this paper is organized as follows. In Sect. 2, some a priori estimates and a sufficient condition for the existence for positive solutions are established (see [1]). In Sect. 3, we study the effect of the small parameter $k_{1}$ and sufficiently large $k_{2}$ in B-D functional response, which proves that the model has at least two positive solutions. In Sect. 4, we investigate the case of sufficiently large $k_{1}$. The results show that if $k_{1}$ is sufficiently large, then the positive solution of this model is determined by a limiting equation. In Sect. 5, we present an asymptotic behavior of solutions depending on the change of time by comparison principle and persistence theorem. Finally, we present a brief summary of this paper.

\section{Preliminaries and lemmas}

The goal of this section is to get a priori upper and lower positive bounds for positive solutions of (3) by using the maximum principle and give some important lemmas. By a method similar to [1], we denote the principal eigenvalue of the following problems by $\lambda_{1}$ and $\mu_{1}$, respectively:

$$
\begin{aligned}
& \begin{cases}\Delta \phi+\lambda \phi f(z, 0)=0, & x \in \Omega, \\
\frac{\partial \phi}{\partial n}+r \phi=0, & x \in \partial \Omega,\end{cases} \\
& \begin{cases}\Delta \psi+\mu \psi g(z, 0)=0, & x \in \Omega, \\
\frac{\partial \psi}{\partial n}+r \psi=0, & x \in \partial \Omega .\end{cases}
\end{aligned}
$$

By $\phi_{1}(x)$ and $\psi_{1}(x)$ we denote the principal eigenfunction such that $\left\|\phi_{1}\right\|=\left\|\psi_{1}\right\|=1$.

For (3), setting $v=0$ or $u=0$, respectively, it is easy to get the following two single species equations:

$$
\begin{aligned}
& \begin{cases}d \Delta u+a(1-q) u f_{1}(z-u, u)=0, & x \in \Omega, \\
\frac{\partial u}{\partial n}+r u=0, & x \in \partial \Omega,\end{cases} \\
& \begin{cases}d \Delta v+b v g(z-v, v)=0, & x \in \Omega, \\
\frac{\partial v}{\partial n}+r v=0, & x \in \partial \Omega .\end{cases}
\end{aligned}
$$

By [1], for (6), we can directly get the following conclusions. 
Lemma 2.1 If $a \leq \frac{\lambda_{1} d}{1-q}$, then 0 is the unique and nonnegative solution of (6); and if $a>\frac{\lambda_{1} d}{1-q}$, then (6) has a unique positive solution, denoted by $\Theta$, satisfying the following properties:

(i) $0<\Theta<z$;

(ii) $\Theta$ is continuously differentiable for $a \in\left(\frac{\lambda_{1} d}{1-q},+\infty\right)$ and is pointwise increasing as a increases;

(iii) $\lim _{a \rightarrow \frac{\lambda_{1} d}{1-q}} \Theta=0$ uniformly for $x \in \bar{\Omega}$, and $\lim _{a \rightarrow \infty} \Theta=z(x)$ for almost every $x \in \Omega$;

(iv) Let $L_{(a, d)}=d \Delta+a(1-q)\left(f(z-\Theta, \Theta)-\Theta f_{1}^{\prime}(z-\Theta, \Theta)+\Theta f_{2}^{\prime}(z-\Theta, \Theta)\right)$ be the linearized operator of (6) at $\Theta$. Then $L_{(a, d)}$ is differentiable in $C_{B}^{2}(\bar{\Omega})=\left\{u \in C^{2}(\bar{\Omega}): \frac{\partial u}{\partial n}+r u=0\right\}$, and all eigenvalues of $L_{(a, d)}$ are strictly negative.

Remark 2.1 For (7), we have the same conclusion as in Lemma 2.1. Suppose that $b>d \mu_{1}$ and denote the unique positive solution by $\theta$. Let $L_{(b, d)}=d \Delta+b\left(g(z-\theta, \theta)-\theta g_{1}^{\prime}(z-\theta, \theta)+\right.$ $\left.\theta g_{2}^{\prime}(z-\theta, \theta)\right)$ be the linearized operator of (7) at $\theta$. Then all eigenvalues of $L_{(b, d)}$ are strictly negative.

Let $\hat{\lambda}_{1}$ be the principal eigenvalue of the equation

$$
\begin{cases}\Delta \phi+\hat{\lambda} \phi f(z-\theta, 0)=0, & x \in \Omega, \\ \frac{\partial \phi}{\partial n}+r \phi=0, & x \in \partial \Omega\end{cases}
$$

and denote the corresponding eigenfunction by $\hat{\phi}_{1}(x)$, which is uniquely determined by the normalization $\left\|\hat{\phi}_{1}\right\|=1$.

Next, we consider the boundary value problem

$$
\begin{cases}d \Delta v+b v g(z-v, v)+a q \Theta f(z-v, 0)=0, & x \in \Omega, \\ \frac{\partial v}{\partial n}+r v=0, & x \in \partial \Omega .\end{cases}
$$

It is easy to get the following results by the method of [1], so we omit the proof.

Lemma 2.2 Suppose $a>\frac{\lambda_{1} d}{1-q}$. Then (9) has the unique positive solution $\hat{v}$, and $0<\hat{v}<z$. In particular, if $b>d \mu_{1}$, then $\theta<\hat{v}<z$.

Theorem 2.1 Suppose that $(u, v)$ is nonnegative solution of (3) and $u \neq \equiv 0, v \neq \equiv$. Then (i) $0<u<\Theta<z, 0<v \leq \hat{v}<z, x \in \bar{\Omega}$; (ii) $u+v<z, x \in \bar{\Omega}$; (iii) $a>\frac{\lambda_{1} d}{1-q}$.

Next, we give the fixed point index of (3) by using the standard fixed point index theory in cone.

We first set up the fixed point index theory for later use. Let $E$ be a Banach space. A set $W \subset E$ is called a wedge if $W$ is a closed convex set and $\alpha W \subset W$ for all $\alpha \geq 0$. For $y \in W$, we define $W_{y}=\{x \in E: \exists r=r(x)>0$ s.t. $y+r x \in W\}$ and $S_{y}=\left\{x \in \bar{W}_{y}:-x \in \bar{W}_{y}\right\}$, and we always assume that $E=\overline{W-W}$. Let $T: W_{y} \rightarrow W_{y}$ be a compact linear operator on $E$. We say that $T$ has property $\alpha$ on $\bar{W}_{y}$ if there exist $t \in(0,1)$ and $\omega \in \bar{W}_{y} \backslash S_{y}$ such that $\omega-t T \omega \in S_{y}$.

Suppose that $F: W \rightarrow W$ is a compact operator and $y_{0} \in W$ is an isolated fixed point of $F$ such that $F y_{0}=y_{0}$. Let $L=F^{\prime}\left(y_{0}\right)$ be the Fréchet derivative at $y_{0}$. Then $L: \bar{W} \rightarrow \bar{W}$. 
Proposition 2.1 (Dancer index theorem [25]) Assume that I- L is invertible on E. Then we have:

(i) If $L$ has property $\alpha$ on $\bar{W}$, then $\operatorname{index}_{W}\left(F, y_{0}\right)=0$;

(ii) If $L$ does not have property $\alpha$ on $\bar{W}$, then index $\operatorname{de}_{W}\left(F, y_{0}\right)=\operatorname{index}_{E}(L, \theta)=(-1)^{\sigma}$, where $\sigma$ is the sum of multiplicities of all eigenvalues of $L$ greater than one.

Proposition 2.2 ([25]) Assume that $F(\theta)=\theta$, and let $A_{0}=F^{\prime}(\theta)$ be the Fréchet derivative of $F$ at $\theta$ in $W$. If the eigenvalue problem

$$
A_{0} h=\lambda h, \quad h \in W
$$

has no eigenvalue equal to 1 , then $\theta$ is an isolated fixed point of $F$, and

(i) if (10) has no eigenvalue greater than 1 , then $\operatorname{index}_{W}(F, \theta)=1$, and

(ii) if (10) has an eigenvalue $\lambda>1$, then $\operatorname{index}_{W}(F, \theta)=0$.

Let $C_{0}(\bar{\Omega})=\left\{y \in C(\bar{\Omega}) \mid \frac{\partial y}{\partial n}+r y=0\right\}$ and $E=\left[C_{0}(\bar{\Omega})\right] \times\left[C_{0}(\bar{\Omega})\right]$. For a sufficiently large $P>0$ and $\tau \in[0,1]$, we consider the following equations:

$$
\left\{\begin{aligned}
(-d \Delta+\tau P) u= & \tau(P+a(1-q) f(z-u-v, u)) u, & & x \in \Omega, \\
(-d \Delta+\tau P) v= & \tau(P v+b g(z-u-v, v) v & & \\
& +q a u f(z-u-v, u)), & & x \in \Omega, \\
\frac{\partial u}{\partial n}+r u=0, & \frac{\partial v}{\partial n}+r v=0, & & x \in \partial \Omega .
\end{aligned}\right.
$$

For $(u, v)^{T} \in E$ and $\tau \in[0,1],(U, V)^{T} \in\left[C^{1+\alpha}(\bar{\Omega})\right] \times\left[C^{1+\alpha}(\bar{\Omega})\right]$ is the unique solution of the following linear problem:

$$
\begin{cases}(-d \Delta+\tau P) U=\tau(P+a(1-q) f(z-u-v, u)) u, & x \in \Omega, \\ (-d \Delta+\tau P) V=\tau(P v+b g(z-u-v, v) v+q a u f(z-u-v, u)), & x \in \Omega, \\ \frac{\partial U}{\partial n}+r U=0, \quad \frac{\partial V}{\partial n}+r V=0, & x \in \partial \Omega .\end{cases}
$$

Define $F_{\tau}:[0,1] \times E \rightarrow E$ and $F_{\tau}(u, v)^{T}=(U, V)^{T}$. It follows from [13] that $F_{\tau}$ is compact. It is clear that $(u, v)^{T} \in E$ is a fixed point of $F_{\tau}$ if and only if $(u, v)^{T} \in E$ is a positive solution of (3). Let

$$
\left\{\begin{array}{l}
K_{0}=\left\{u \in C_{0}(\bar{\Omega}) \mid u(x) \geq 0, x \in \Omega\right\} \\
W=\{(u, v) \in E \mid u(x) \geq 0, v(x) \geq 0, x \in \Omega\} \\
D=\left\{(u, v) \in W \mid 0 \leq u(x) \leq \Theta, 0 \leq v(x) \leq \max _{\bar{\Omega}} \hat{v}+1, x \in \Omega\right\} \\
D^{\prime}=(\operatorname{int} D) \cap W
\end{array}\right.
$$

Then $W$ is a cone in $E$, and $D$ is bounded set in $W$. Let $F=F_{1}$. Then there exists $K>0$ such that $f(z-u-v, u) \geq f(z-u, u)-K v$.

Suppose that $P$ is sufficiently large such that, for all $(u, v) \in D$,

$$
P+a(1-q) f(z-u-v, u)>0, \quad P+b g(z-u-v, v)-a q u K>0 .
$$


Then $F: D \rightarrow W$ is continuously differentiable, and hence (3) has nonnegative solutions if only and if $F$ has a fixed point on $D$. According to the homotopy invariance of degree, we have $\operatorname{deg}_{W}\left(I-F_{\tau}, D^{\prime},(0,0)\right)=\operatorname{deg}_{W}\left(I-F, D^{\prime},(0,0)\right), \tau \in[0,1]$. It follows that the index numbers of $(0,0)$ and $(0, \theta)$ are well defined by using the fixed point theory as the following lemmas.

Lemma 2.3 ([1]) The index number of the operator $F$ at $(0,0)$ has the following properties:

(i) if $a \neq \frac{\lambda_{1} d}{1-q}, b>\mu_{1} d$, then $\operatorname{index}_{W}(F,(0,0))=0$;

(ii) Suppose that $b<\mu_{1} d$. If $a>\frac{\lambda_{1} d}{1-q}$, then $\operatorname{index}_{W}(F,(0,0))=0$; If $a<\frac{\lambda_{1} d}{1-q}$, then $\operatorname{index}_{W}(F,(0,0))=1$;

(iii) $\operatorname{index}_{W}\left(F, D^{\prime}\right)=1$.

Lemma 2.4 ([1]) Suppose $b>\mu_{1} d$. The index at the point $(0, \theta)$ has the following properties:

(i) If $a<\frac{\hat{\lambda}_{1} d}{1-q}$, then $\operatorname{index}_{W}(F,(0, \theta))=1$; if $a>\frac{\hat{\lambda}_{1} d}{1-q}$, then $\operatorname{index}_{W}(F,(0, \theta))=0$;

(ii) If $a=\frac{\hat{\lambda}_{1} d}{1-q}$, then either (3) has a positive solution, or $\operatorname{index}_{W}(F,(0, \theta))=1$.

Combining with the previous lemma, according to [1], we can show the following sufficient condition for the existence of nonnegative solutions to equation (3).

\section{Theorem 2.2}

(i) If $a<\frac{\lambda_{1} d}{1-q}, b<\mu_{1} d$, then 0 is the only nonnegative solution of (3);

(ii) If $a>\frac{\lambda_{1} d}{1-q}, b<\mu_{1} d$, then (3) has at least one positive solution besides the zero solution;

(iii) If $a>\frac{\hat{\lambda}_{1} d}{1-q}, b>\mu_{1} d$, then (3) has at least one positive solution besides $(0,0)$ and $(0, \theta)$.

\section{The effect of mutual interference between predators}

In this section, we investigate the multiplicity and stability of positive solutions of system (3) under the effect of the parameters $k_{i}(i=1,2)$ by the standard perturbation theory.

According to Theorems 2.1 and 2.2, sufficient conditions for the existence of positive solutions of (3) are $b>\mu_{1} d$ and $a>\frac{\hat{\lambda}_{1} d}{1-q}$, and a necessary condition is $a>\frac{\lambda_{1} d}{1-q}$; moreover, $\frac{\lambda_{1} d}{1-q}<\frac{\hat{\lambda}_{1} d}{1-q}$. Next, we study the multiplicity and stability of positive solutions of (3) when $k_{1}$ is small enough and $k_{2}$ is sufficiently large with $q>\frac{1}{2}$.

In [26], taking $a$ as a bifurcation parameter and using the local bifurcation theory, we get that the positive solution $(u(s), v(s))$ bifurcates from the semitrivial solution $(0, \theta)$. According to Lemma 2.4.9 in [26], we will show that system (3) has at least one positive solution besides the bifurcation solution $(u(s), v(s))$ when $k_{1}$ is small enough and $k_{2}$ is sufficiently large with $q>\frac{1}{2}$. Then we can establish the following result.

Theorem 3.1 Suppose that $b>\mu_{1} d$ and $q>\frac{1}{2}$. If there exist sufficiently large $K_{2}>0$ and suitably large $D>0$ such that $k_{2}>K_{2}, d>D$, and small enough $k_{1}$, then the local bifurcation of the positive solution $(u(s), v(s))$ is nondegenerate and unstable for $a \in\left(\frac{\hat{\lambda}_{1} d}{1-q}-\varepsilon, \frac{\hat{\lambda}_{1} d}{1-q}\right)$ with $\varepsilon>0$; moreover, (3) has at least two positive solutions.

Proof Firstly, we prove that any positive solution bifurcated from $(0, \theta)$ is nondegenerate and unstable. To complete this, we need only show that there exists a sufficiently small $\varepsilon>0$ 
such that any positive solution $(u(s), v(s))$ of (3) is nondegenerate for $a \in\left(\frac{\hat{\lambda}_{1} d}{1-q}-\varepsilon, \frac{\hat{\lambda}_{1} d}{1-q}\right)$, and the corresponding linearized eigenvalue problem

$$
\begin{cases}d \Delta \xi+a(1-q) \xi h_{1}(u(s), v(s)) & \\ \quad-a(1-q) u(s) f_{1}^{\prime}(z-u(s)-v(s), u(s)) \eta+\gamma \xi=0, & x \in \Omega, \\ d \Delta \eta+b h_{2}(u(s), v(s)) \eta-q a u(s) f_{1}^{\prime}(z-u(s)-v(s), u(s)) \eta & \\ -b v(s) g_{1}^{\prime}(z-u(s)-v(s), v(s)) \xi+a q h_{1}(u(s), v(s)) \xi+\gamma \eta=0, & x \in \Omega,\end{cases}
$$

where

$$
\begin{aligned}
h_{1}(u(s), v(s))= & f(z-u(s)-v(s), u(s)) \\
& -u(s) f_{1}^{\prime}(z-u(s)-v(s), u(s))+u(s) f_{2}^{\prime}(z-u(s)-v(s), u(s)), \\
h_{2}(u(s), v(s))= & g(z-u(s)-v(s), v(s)) \\
& -v(s) g_{1}^{\prime}(z-u(s)-v(s), v(s))+v(s) g_{2}^{\prime}(z-u(s)-v(s), v(s)),
\end{aligned}
$$

has a unique eigenvalue $\hat{\gamma}$ such that $\operatorname{Re}(\hat{\gamma})<0$ with multiplicity one. Set the sequences $\left\{s_{n}\right\}$ and $\left\{a_{n}\right\}$. Then $u(s)=u_{n}(s):=u_{n}, v(s)=v_{n}(s):=v_{n}$ are the positive solution of (3) with $k_{1}=k_{1, n}$ and $a=a_{n}$. Denote the corresponding eigenvalue by $\gamma=\gamma_{n}$. By the assumption we can suppose that, as $n \rightarrow \infty, \varepsilon_{n}>0$ and $\varepsilon_{n} \rightarrow 0, k_{1, n}>0$ and $k_{1, n} \rightarrow 0, a_{n} \in\left(\frac{\hat{\lambda}_{1} d}{1-q}-\varepsilon, \frac{\hat{\lambda}_{1} d}{1-q}\right)$. If $n \rightarrow \infty$, then $k_{1, n} \rightarrow 0, \gamma_{n} \rightarrow \gamma, a_{n} \rightarrow \frac{\hat{\lambda}_{1} d}{1-q}$, and $\left(u_{n}, v_{n}\right) \rightarrow(u(0), v(0))=(0, \theta)$. Hence, the linearized problem (12) converges to the following problem:

$$
\begin{cases}d \Delta \xi+a(1-q) \xi f(z-\theta, 0)+\gamma \xi=0, & x \in \Omega, \\ d \Delta \eta+L_{b} \eta-b \theta g_{1}^{\prime}(z-\theta, \theta) \xi+a q f(z-\theta, 0) \xi+\gamma \eta=0, & x \in \Omega\end{cases}
$$

where $L_{b}=b\left[g(z-\theta, \theta)-\theta g_{1}^{\prime}(z-\theta, \theta)+\theta g_{2}^{\prime}(z-\theta, \theta)\right]$. It is easy to see that 0 is a simple eigenvalue of (12) with the corresponding eigenfunction $(\xi, \eta)=\left(\hat{\phi}_{1}, \chi_{1}\right)$, where $\chi_{1}=$ $L_{(b, d)}^{-1}\left(\left(b \theta g_{1}^{\prime}(z-\theta, \theta)+a q f(z-\theta, 0)\right) \hat{\phi}_{1}\right)$. Moreover, all other eigenvalues are positive and stand apart from 0 . By the eigenvalue perturbation theory[25] we get that problem (12) has a unique eigenvalue $\gamma_{n} \rightarrow 0$ for large $n$ with multiplicity one, and real parts of all other eigenvalues are positive and stand apart from 0 . We may assume that $\left(\xi_{n}, \eta_{n}\right)$ is the corresponding eigenfunction to $\gamma_{n}$. Then $\left(\xi_{n}, \eta_{n}\right) \rightarrow\left(\hat{\phi}_{1}, \chi_{1}\right)$ as $n \rightarrow \infty$. We further show that $\operatorname{Re} \gamma_{n}<0$ for $n$ large enough. Multiplying the first equation of (12) with the sequence $n$ by $\hat{\phi}_{1}$ and integrating on $\Omega$, we have

$$
\begin{aligned}
& -d \int_{\Omega} \hat{\phi}_{1} \Delta \xi_{n}-a_{n}(1-q) \int_{\Omega}\left[f\left(z-u_{n}-v_{n}, u_{n}\right)\right. \\
& \left.\quad-u_{n} f_{1}^{\prime}\left(z-u_{n}-v_{n}, u_{n}\right)+u_{n} f_{2}^{\prime}\left(z-u_{n}-v_{n}, u_{n}\right)\right] \xi_{n} \hat{\phi}_{1} \\
& \quad+a_{n}(1-q) \int_{\Omega} u(s) f_{1}^{\prime}(z-u(s)-v(s), u(s)) \eta_{n} \\
& =\int_{\Omega} \gamma_{n} \hat{\phi}_{1} \xi_{n} .
\end{aligned}
$$


Taking $(a, u, v)=\left(a_{n}, u_{n}, v_{n}\right)$ in the first equation of (3), multiplying by $\xi_{n}$, and integrating on $\Omega$, we obtain

$$
-d \int_{\Omega} \hat{\phi}_{1} \Delta \xi_{n}-a_{n}(1-q) \int_{\Omega} u_{n} f\left(z-u_{n}-v_{n}, u_{n}\right) \xi_{n} .
$$

By (14)-(15), $u_{n}(s)=\left(s_{n} \hat{\phi}_{1}+O\left(s_{n}^{2}\right)\right), v_{n}(s)=\theta-s_{n} \chi_{1}+O\left(s_{n}^{2}\right)$ (see Theorem 2.4.1 in [26]). Simplifying and letting $n \rightarrow \infty$, we have

$$
\lim _{n \rightarrow \infty} \frac{\gamma_{n}}{s_{n}}=\frac{d \hat{\lambda}_{1} \int_{\Omega}\left[\left(\hat{\phi}_{1}-\chi_{1}\right) f_{1}^{\prime}(z-\theta, 0)-f_{2}^{\prime}(z-\theta, 0) \hat{\phi}_{1}\right] \hat{\phi}_{1}^{2} d x}{(1-q) \int_{\Omega} f(z-\theta, 0) \hat{\phi}_{1}^{2} d x} .
$$

Since $\hat{\phi}_{1}, \chi_{1}$ satisfy the equations

$$
\left\{\begin{array}{l}
d \Delta \hat{\phi}_{1}+\hat{\lambda}_{1} d f(z-\theta, 0) \hat{\phi}_{1}=0, \\
d \Delta \chi_{1}+b\left[g(z-\theta, \theta)-\theta g_{1}^{\prime}(z-\theta, \theta)+\theta g_{2}^{\prime}(z-\theta, \theta)\right] \chi_{1} \\
\quad+q \frac{\hat{\lambda}_{1} d}{1-q} f(z-\theta, 0) \hat{\phi}_{1}-b \theta g_{1}^{\prime}(z-\theta) \hat{\phi}_{1}=0,
\end{array}\right.
$$

subtracting the first equation from the second equation, we obtain

$$
\begin{aligned}
& {\left[d \Delta\left(\chi_{1}-\hat{\phi}_{1}\right)+b\left[g(z-\theta, \theta)-\theta g_{1}^{\prime}(z-\theta, \theta)+\theta g_{2}^{\prime}(z-\theta, \theta)\right]\right]\left(\chi_{1}-\hat{\phi}_{1}\right)} \\
& \quad=-\left[b \left[g(z-\theta, \theta)-2 \theta g_{1}^{\prime}(z-\theta, \theta)+\theta .\right.\right.
\end{aligned}
$$

By Remark 2.1 we know that all eigenvalues of $L_{(b, d)}=d \Delta\left(\chi_{1}-\hat{\phi}_{1}\right)-b\left[g(z-\theta, \theta)+\theta g_{1}^{\prime}(z-\right.$ $\left.\theta, \theta)+\theta g_{2}^{\prime}(z-\theta, \theta)\right]$ are strictly negative, and thus $L_{(b, d)}$ is a reversible negative operator. Set

$$
\begin{aligned}
\hat{T} & =-\left\{b\left[g(z-\theta, \theta)-2 \theta g_{1}^{\prime}(z-\theta, \theta)+\theta g_{2}^{\prime}(z-\theta)\right]+\frac{(2 q-1) \lambda^{\prime} d}{1-q} f(z-\theta, 0)\right\} \hat{\phi}_{1} \\
& =-\left\{\frac{b\left[(z-\theta)\left(1+m_{2}(z-\theta)-2 \theta\left(1+k_{2} \theta\right)\right]\right.}{\left[1+m_{2}(z-\theta)+k_{2} \theta\right]^{2}}+\frac{(2 q-1) \hat{\lambda}_{1} d}{1-q} f(z-\theta, 0)\right\} \hat{\phi}_{1} .
\end{aligned}
$$

Then

$$
\chi_{1}-\hat{\phi}_{1}=L_{(b, d)}^{-1} \hat{T} .
$$

Obviously, as $q>\frac{1}{2}$, if there exist sufficiently large $K_{2}>0$ and suitable large $D>0$ such that $k_{2}>K_{2}$ and $d>D$, then $\hat{\phi}_{1}-\chi_{1}<0$, and if $k_{1}$ is small enough, then $f_{2}^{\prime}(z-\theta, 0) \hat{\phi}_{1}$ is sufficiently small, and hence $\gamma_{n}^{\prime}(0)<0$ as $n \gg 1$. Thus, if $k_{2}>K_{2}$ and $d>D$, then there exists sufficiently large $N$ such that $\operatorname{Re} \gamma_{n}<0$ for $n>N$.

Next, to prove that there exists at least two positive solutions, we may use apagoge and suppose that (3) has a unique positive solution $(\tilde{u}, \tilde{v})$. Then it follows from local bifurcation theory that it must be a positive solution bifurcated from near $(0, \theta)$; moreover, $(\tilde{u}, \tilde{v})$ is nondegenerate, and the corresponding linearized eigenvalue problem has a unique eigenvalue $\tilde{\gamma}$ such that $\operatorname{Re} \tilde{\gamma}<0$ with multiplicity one. By all these facts it is easy to see that $I-F^{\prime}(\tilde{u}, \tilde{v})$ is invertible and $F^{\prime}(\tilde{u}, \tilde{v})$ does not have property $\alpha$ on $\bar{W}_{(\tilde{u}, \tilde{v})}$, and then 
$\operatorname{index}_{W}(F,(\tilde{u}, \tilde{v}))=(-1)^{\sigma}$, where $\sigma$ is the sum of multiplicities of all the eigenvalues of $F^{\prime}(\tilde{u}, \tilde{v})$ that are greater than one; obviously, $\sigma=1$. Thus $\operatorname{index}_{W}(F,(\tilde{u}, \tilde{v}))=(-1)^{1}=-1$. Using Lemmas 2.3-2.4 and the additivity property of the fixed point index, we obtain

$$
1=\operatorname{index}_{W}\left(F, D^{\prime}\right)=\operatorname{index}_{W}(F,(0,0))+\operatorname{index}_{W}(F,(0, \theta))+\operatorname{index}_{W}(F,(\tilde{u}, \tilde{v}))=0,
$$

which gives a contradiction, and the proof is completed.

Remark 3.1 Theorem 2.4.3 in [26] shows that, as $a>\frac{\hat{\lambda}_{1} d}{1-q}$, the bifurcation solution extends to $\infty$ by $a$. However, Theorem 3.1 indicates that, as $k_{1}$ is small enough and $k_{2}$ sufficiently large, $d$ is suitably large, and $q>\frac{1}{2}$, then $a=a(s) \in\left(\frac{\lambda_{1} d}{1-q}, \frac{\hat{\lambda}_{1} d}{1-q}\right)$. Then there exists $a^{*} \in\left(\frac{\lambda_{1} d}{1-q}, \frac{\hat{\lambda}_{1} d}{1-q}\right)$ such that (3) has at least two solutions for $a \in\left(a^{*}, \frac{\hat{\lambda}_{1} d}{1-q}\right)$.

\section{The effect of $k_{1}$ on uniqueness and stability}

In this section, we consider the effect of $k_{1}$ on the existence, uniqueness, and stability of positive solutions of (3) as $k_{1} \rightarrow \infty$.

Firstly, we can get that any positive solution $(u, v)$ of (3) satisfies the following result: as $k_{1}$ is sufficiently large, $k_{1} u$ converges to the positive solution of the following problem:

$$
\begin{cases}\Delta w+a(1-q) w \hat{f}(z-\theta, w)=0, & x \in \Omega, \\ \frac{\partial w}{\partial n}+r w=0, & x \in \partial \Omega,\end{cases}
$$

where $\hat{f}(z-\theta, w)=\frac{z-\theta}{1+m_{1}(z-\theta)+w}$, which implies that equation (16) almost determines all positive solutions of (3) when $k_{1}$ is sufficiently large.

Next, we investigate the uniqueness of positive solutions of (16).

Lemma 4.1 Problem (16) has one positive solution $w_{0}$ if and only if $a>\frac{d \hat{\lambda}_{1}}{1-q}$. In addition, the positive solution is unique and asymptotically stable.

Proof Let $w$ be a positive solution of (16). Then

$$
a(1-q)=d \lambda_{1}(\hat{f}(z-\theta, w))>d \lambda_{1}(\hat{f}(z-\theta, 0))=d \lambda_{1}(f(z-\theta, 0))=d \hat{\lambda}_{1} .
$$

It follows that $a>\frac{\hat{\lambda}_{1} d}{1-q}$. Conversely, if $a>\frac{\hat{\lambda}_{1} d}{1-q}$, then we may show that (16) has a unique positive solution. Firstly, we prove that there exists a constant $M>0$ such that all positive solutions $w$ of (16) satisfy $\|w\|_{C^{1}} \leq M$ when $a>\frac{\hat{\lambda}_{1} d}{1-q}$. Otherwise, if this proposition does not hold, then suppose that $a=a_{i}$ and $a_{i} \rightarrow a \geq \frac{d \hat{\lambda}_{1}}{1-q}$ as $i \rightarrow \infty$. It follows that $w_{i}$ is the positive solution of (16) and $\left\|w_{i}\right\|_{\infty} \rightarrow \infty, \hat{f}\left(z-\theta, w_{i}\right)=\frac{z-\theta}{1+m_{1}(z-\theta)+w_{i}} \stackrel{L^{2}}{\rightarrow} h_{1}$.

Let $\bar{w}_{i}=w_{i} /\left\|w_{i}\right\|_{\infty}$. Then

$$
\begin{cases}\Delta \bar{w}_{i}+a(1-q) \bar{w} \hat{f}\left(z-\theta, w_{i}\right)=0, & x \in \Omega, \\ \frac{\partial \bar{w}_{i}}{\partial n}+r \bar{w}_{i}=0, & x \in \partial \Omega .\end{cases}
$$


By using the standard elliptic regularization theory we have that $\bar{w}_{i} \stackrel{C^{1}}{\rightarrow} \bar{w} \geq 0, \not \equiv 0$, and $\bar{w}$ satisfies

$$
\begin{cases}\Delta \bar{w}+a(1-q) \bar{w} h_{1}=0, & x \in \Omega, \\ \frac{\partial \bar{w}}{\partial n}+r \bar{w}=0, & x \in \partial \Omega .\end{cases}
$$

Since $0 \leq h_{1} \leq \frac{1}{k_{1}}$, applying the strong extreme value theory and the Hopf lemma, we have $\bar{w}>0, x \in \bar{\Omega}$, and then $\hat{f}\left(z-\theta, w_{i}\right)=\frac{z-\theta}{1+m_{1}(z-\theta)+\bar{w}_{i}\left\|w_{i}\right\|_{\infty}} \stackrel{L^{2}}{\longrightarrow} 0$; thereby $h_{1}=0$, and

$$
\begin{cases}\Delta \bar{w}=0, & x \in \Omega \\ \frac{\partial \bar{w}}{\partial n}+r \bar{w}=0, & x \in \partial \Omega\end{cases}
$$

Hence $\bar{w} \equiv 0$, which contracts to $\left\|\bar{w}_{i}\right\|_{\infty}=1$. Thus, we obtain a priori estimates of $w$.

In the following part, we establish the stability and uniqueness of positive solutions of (16). Set $\tilde{P}=\left\{w \in C^{1}(\Omega) \mid w \geq 0, \frac{\partial w}{\partial n}+r w=0\right\}$ and $\tilde{D}=\left\{w \in \tilde{P} \mid\left\|\bar{w}_{i}\right\|_{\infty} \leq M+1\right\}$. Define $B_{t}: \tilde{P} \rightarrow \tilde{P}$ by $B_{t}(w)=\left(-\Delta+M_{1}\right)^{-1}\left(\operatorname{ta}(1-q) w \hat{f}(z-\theta, w)+M_{1} w\right)$, where $M_{1}$ is a positive constant such that $t a(1-q) \hat{f}(z-\theta, w)+M_{1}>0$ for all $w \in \tilde{D}, t \in[0,1]$. Then $B_{t}$ is a compact operator. Assume that $B=B_{1}$. Then $B: \tilde{P} \rightarrow \tilde{P}$ is continuous and differentiable; moreover, system (16) has a nonnegative solution if and only if the operator $B$ has one fixed point in $\tilde{D}$. Applying the method similar to that of Lemma 2.3.2 in [26], we have index $\tilde{P}(B, \tilde{D})=1$ and $\operatorname{index}_{\tilde{P}}(B, 0)=0$. Hence, there exists sufficiently small $\epsilon$ such that

$$
\operatorname{index}_{\tilde{P}}\left(B, \tilde{D} \backslash B_{\epsilon}\right)=\operatorname{index}_{\tilde{P}}(B, \tilde{D})-\operatorname{index}_{\tilde{P}}(B, 0)=1 .
$$

Thus, (16) has at least one positive solution when $a>\frac{d \hat{\lambda}_{1}}{1-q}$.

We investigate the following eigenvalue problem:

$$
\begin{cases}-\Delta \chi-a(1-q) \chi\left[\hat{f}(z-\theta, w)+w \hat{f}_{2}^{\prime}(z-\theta, w)\right]=\eta \chi, & x \in \Omega, \\ \frac{\partial \chi}{\partial n}+r \chi=0, & x \in \partial \Omega,\end{cases}
$$

where $w$ is a positive solution of (16). Applying the comparison principle of eigenvalues, we have

$$
\eta_{1}=d \lambda_{1}\left(-a(1-q) \chi\left[\hat{f}(z-\theta, w)+w \hat{f}_{2}^{\prime}(z-\theta, w)\right]\right)>d \lambda_{1}(-a(1-q) \chi \hat{f}(z-\theta, w))=0 .
$$

Therefore, (17) has no eigenvalues less than 0 when $a>\frac{d \hat{\lambda}_{1}}{1-q}$. So any positive solution of (16) is nondegenerate and asymptotically stable, and $\operatorname{index}_{\tilde{P}}(B, w)=(-1)^{0}=1$, which implies that (16) has at most finitely many positive solutions, which we denote by $\left\{w_{i}, 1 \leq i \leq l\right\}$. Using fixed point index theory, the number of positive solutions of equation (16) can be calculated as follows:

$$
k=\operatorname{index}_{\tilde{P}}(B, 0)+\sum_{i=1}^{l} \operatorname{index}_{\tilde{P}}\left(B, w_{i}\right)=\operatorname{index}_{\tilde{P}}(B, \tilde{D})=1,
$$

and, as a result, equation (16) has a unique positive solution. 
Theorem 4.1 Suppose that $b>d \mu_{1}$ is a fixed constant. For any small $\varepsilon>0$ and any $A>\frac{d \hat{\lambda}_{1}}{1-q}$, there exists sufficiently large $K_{1}=K_{1}(\varepsilon, A)>0$ such that any positive solution of (3) satisfies $\|u\|_{c_{1}}+\|v-\theta\|_{c_{1}} \leq \varepsilon$ when $k_{1}>K_{1}$. In particular, if we choose a sufficiently large $K_{1}(\varepsilon, A)$ such that $k_{1}>K_{1}$ and $a \in\left(\frac{d \hat{\lambda}_{1}}{1-q}, A\right]$, then $\left\|k_{1} u-w_{0}\right\|_{c_{1}} \leq \varepsilon$, where $w_{0}$ is the unique positive solution of (16).

Proof If the first part of the conclusion is false, then there are $A_{0}>\frac{d \hat{\lambda}_{1}}{1-q}, k_{1, i} \rightarrow \infty, a_{i} \in$ $\left(\frac{d \hat{\lambda}_{1}}{1-q}, A_{0}\right]$, such that the positive solution $\left(u_{i}, v_{i}\right)$ of (3) is bounded away the semitrivial $(0, \theta)$ with $a=a_{i}, k_{1}=k_{1, i}$. Since $0<u_{i}+v_{i}<z,\left\{-\Delta u_{i}\right\}$ and $\left\{-\Delta v_{i}\right\}$ of (3) are bounded on $L^{\infty}(\bar{\Omega})$. By the $L^{p}$ estimates and embedding theorem of elliptic equation, we may assume that $a_{i} \rightarrow a \in\left(\frac{d \hat{\lambda}_{1}}{1-q}, A_{0}\right], u_{i} \stackrel{C^{1}}{\rightarrow} u \geq 0, v_{i} \stackrel{C^{1}}{\rightarrow} v \geq 0$, where $u, v \in C_{B}^{1}(\bar{\Omega})$. Furthermore, $f\left(z-u_{i}-\right.$ $\left.v_{i}, u_{i}\right)=\frac{z-u_{i}-v_{i}}{1+m_{1}\left(z-u_{i}-v_{i}\right)+k_{1, i} u_{i}} \stackrel{L^{2}}{\rightarrow} h_{2}$ with $0 \leq h_{2} \leq 0$, and $u$ satisfies

$$
\begin{cases}d \Delta u+a(1-q) u h_{2}=0, & x \in \Omega, \\ \frac{\partial u}{\partial n}+r u=0, & x \in \partial \Omega .\end{cases}
$$

Suppose $u \equiv 0$. Then $v$ satisfies

$$
\begin{cases}d \Delta v+b v g(z-v, v)=0, & x \in \Omega, \\ \frac{\partial v}{\partial n}+r v=0, & x \in \partial \Omega,\end{cases}
$$

and $v \equiv 0$ or $v=\theta$ as $b>d \mu_{1}$. If $v \equiv 0$, let $\bar{v}_{i}=v_{i} /\left\|v_{i}\right\|_{\infty}$. Then

$$
\begin{cases}d \Delta \bar{v}_{i}+b \bar{v}_{i} g\left(z-v_{i}, v_{i}\right)=0, & x \in \Omega, \\ \frac{\partial \bar{v}_{i}}{\partial n}+r \bar{v}_{i}=0, & x \in \partial \Omega .\end{cases}
$$

By the standard regularization theory we may assume that $\bar{v}_{i} \stackrel{C^{1}}{\rightarrow} \bar{v}$, and thus the limit equation of this equation is

$$
\begin{cases}d \Delta \bar{v}+b \bar{v} g(z, 0)=0, & x \in \Omega, \\ \frac{\partial \bar{v}}{\partial n}+r \bar{v}=0, & x \in \partial \Omega .\end{cases}
$$

Multiplying (18) by $\psi_{1}$ and integrating on $\Omega$, we easily see that $\int_{\Omega}\left(b-d \mu_{1}\right) \psi_{1} \bar{v} g(z, 0)=0$. Since $b>d \mu_{1}, \psi_{1}>0$, and $g(z, 0)>0$, it follows that $\bar{v} \equiv 0$, which is a contradiction. Hence, $v=\theta,\left(u_{i}, v_{i}\right) \stackrel{C^{1}}{\rightarrow}(0, \theta)$, which contradicts to the assumption that the positive solution $\left(u_{i}, v_{i}\right)$ is depart from $(0, \theta)$.

Suppose $u \geq 0, \not \equiv 0$. By the strong maximum principle we get $u>0$, and thereby

$$
\begin{cases}d \Delta u=0, & x \in \Omega, \\ \frac{\partial u}{\partial n}+r u=0, & x \in \partial \Omega .\end{cases}
$$

We know that $u \equiv 0$, a contradiction.

Next, we prove the last part of the theorem. We only need to show that $k_{1, i} u_{i}$ is near some positive solution of (16) when $a=a_{i}$. We first show that $k_{1, i}\left\|u_{i}\right\|_{\infty}$ is uniformly bounded. 
Otherwise, suppose that $k_{1, i}\left\|u_{i}\right\|_{\infty} \rightarrow \infty$ and let $\bar{u}_{i}=u_{i} /\left\|u_{i}\right\|_{\infty}$. Then

$$
\begin{cases}d \Delta \bar{u}_{i}+a_{i}(1-q) \bar{u}_{i} f\left(z-u_{i}-v_{i}, u_{i}\right)=0, & x \in \Omega, \\ \frac{\partial \bar{u}_{i}}{\partial n}+r \bar{u}_{i}=0, & x \in \partial \Omega .\end{cases}
$$

Applying the standard regularization theory, we may assume that $\bar{u}_{i} \stackrel{C^{1}}{\rightarrow} \bar{u} \geq 0, \not \equiv 0$ in the $C_{B}^{1}, f\left(z-u_{i}-v_{i}, u_{i}\right) \stackrel{L^{2}}{\rightarrow} h_{2}$, and $a_{i} \rightarrow a \in\left(\frac{d \hat{\lambda}_{1}}{1-q}, A_{0}\right]$.

Taking the limit on both sides of (19), we get that $\bar{u}$ is a weak solution satisfying the following equation:

$$
\begin{cases}d \Delta \bar{u}+a(1-q) \bar{u} h_{2}=0, & x \in \Omega, \\ \frac{\partial \bar{u}}{\partial n}+r \bar{u}=0, & x \in \partial \Omega .\end{cases}
$$

By the maximum principle and Hopf lemma we have $\bar{u}>0(x \in \Omega)$. Hence, since $f\left(z-u_{i}-\right.$ $\left.v_{i}, u_{i}\right)=\frac{z-u_{i}-v_{i}}{1+m_{1}\left(z-u_{i}-v_{i}\right)+k_{1, i} \bar{u}_{i}\left\|u_{i}\right\|_{\infty}} \stackrel{L^{2}}{\rightarrow} h_{2}=0$ as $i \rightarrow \infty$, it is easy to see that $\bar{u} \equiv 0(x \in \Omega)$, a contradiction. Thus $k_{1, i}\left\|u_{i}\right\|_{\infty}$ is uniformly bounded.

Let $w_{i}=k_{1, i} u_{i}$, Then $w_{i}$ satisfies

$$
\begin{cases}d \Delta w_{i}+a_{i}(1-q) w_{i} \hat{f}\left(z-u_{i}-v_{i}, w_{i}\right)=0, & x \in \Omega, \\ \frac{\partial w_{i}}{\partial n}+r w_{i}=0, & x \in \partial \Omega .\end{cases}
$$

Since $\left\|w_{i}\right\|_{\infty}$ is bounded, applying the standard regularization theory and the Sobolev embedding theorem, we can obtain that $w_{i} \stackrel{C^{1}}{\rightarrow} w$. Letting $i \rightarrow \infty$, the limit equation of (20) is (16), that is, $w$ is a nonnegative solution of (20). When $a=\frac{d \hat{\lambda}_{1}}{1-q}, w_{i}=k_{1, i} u_{i} \rightarrow w \equiv 0$. If $a_{i} \rightarrow \frac{d \hat{\lambda}_{1}}{1-q}$, then any positive solution of (16) converges to 0 when $a=a_{i}$. Hence, $w_{i}=k_{1, i} u_{i}$ must be near to some positive solution (16) as $a=a_{i}$.

We only need to prove that $w$ is a positive solution of (16) when $a>\frac{d \hat{\lambda}_{1}}{1-q}$. If $w$ is not always a positive solution, then it follows from the maximum principle that $w \equiv 0$. Let $\bar{w}_{i}=w_{i} /\left\|w_{i}\right\|_{\infty}$. Then

$$
\begin{cases}d \Delta \bar{w}_{i}+a_{i}(1-q) \bar{w} \hat{f}\left(z-u_{i}-v_{i}, w_{i}\right)=0, & x \in \Omega, \\ \frac{\partial \bar{w}_{i}}{\partial n}+r \bar{w}_{i}=0, & x \in \partial \Omega .\end{cases}
$$

Suppose $\bar{w}_{i} \stackrel{C^{1}}{\rightarrow} \bar{w}$. By taking the limit of (21) we have

$$
\begin{cases}d \Delta \bar{w}+a_{i}(1-q) \bar{w} \hat{f}(z-\theta, 0)=0, & x \in \Omega, \\ \frac{\partial \bar{w}}{\partial n}+r \bar{w}=0, & x \in \partial \Omega .\end{cases}
$$

Since $\bar{w} \geq 0, \not \equiv 0$, it follows from the maximum principle that $\bar{w}>0$, and thus $a=\frac{d \hat{\lambda}_{1}}{1-q}$, which deduces a contradiction. Hence $w>0$, that is, $k_{1, i} u_{i}$ converges to the unique positive solution $w_{0}$ of (16).

Finally, we consider the existence and stability of positive solutions of (3) when parameter $k_{1}$ is large enough. 
Theorem 4.2 Suppose that $b>d \mu_{1}$. For any small $\varepsilon>0$ and some $A \geq \frac{\hat{\lambda}_{1} d}{1-q}$, there exists sufficiently large $K_{1}=K_{1}(\varepsilon, A)$ such that $k_{1}>K_{1}$. Then we have:

(i) If $a \in\left[\frac{\lambda_{1} d}{1-q}+\varepsilon, \frac{\hat{\lambda}_{1} d}{1-q}\right)$, then (3) has no positive solutions;

(ii) If $a \in\left[\frac{\hat{\lambda}_{1} d}{1-q}, A\right]$, then (3) has the unique positive solution, and it is asymptotically stable.

Proof (i) Suppose that the conclusion is false. Then there exist $\varepsilon_{0}>0, k_{1, i} \rightarrow \infty$, and $a_{i} \rightarrow a \in\left[\frac{d \lambda_{1}}{1-q}+\varepsilon_{0}, \frac{d \hat{\lambda}_{1}}{1-q}\right)$ such that $\left(u_{i}, v_{i}\right)$ is a positive solution of $(3)$ as $\left(a, k_{1}\right)=\left(a_{i}, k_{1, i}\right)$. It follows from Lemma 4.1 that $k_{1, i}\left\|u_{i}\right\|_{\infty}$ is uniformly bounded, Let $w_{i}=k_{1, i} u_{i}$, then $w_{i}$ satisfies equation (21). By the standard regularized theory and the Sobolev embedding theorem we may suppose that $w_{i} \stackrel{C^{1}}{\rightarrow} w$. Hence, $w$ is a nonnegative solution of (16). Since $a \in\left[\frac{d \lambda_{1}}{1-q}+\varepsilon_{0}, \frac{d \hat{\lambda}_{1}}{1-q}\right)$, combining with Theorem 4.1 , we get $w \equiv 0$. Applying a similar method as in the case $a>\frac{d \hat{\lambda}_{1}}{1-q}$ in Theorem 4.1, it is easy to get a contradiction.

(ii) Firstly, we prove that any positive solution of (3) is nondegenerate and linearly stable for condition (ii). Assuming that $(u, v)$ is a positive solution of (3), let $\hat{u}=k_{1} u, m=\frac{1}{k_{1}}$. Then $(u, v)$ is a positive solution of (3) if only and if $\left(k_{1} u, v\right)$ is a solution of the following problem:

$$
\begin{cases}d \Delta \hat{u}+(1-q) a \hat{u} f(z-m \hat{u}-v, \hat{u})=0, & x \in \Omega, \\ d \Delta v+b v g(z-m \hat{u}-v, v)+q a m \hat{u} f(z-m \hat{u}-v, \hat{u})=0, & x \in \Omega, \\ \frac{\partial \hat{u}}{\partial n}+r \hat{u}=0, \quad \frac{\partial v}{\partial n}+r v=0, & x \in \partial \Omega .\end{cases}
$$

Next, we only need to prove the nondegeneracy and stability of (22). We suppose that the conclusion is false. Then there are $\varepsilon_{0}>0, A_{0}>\frac{d \hat{\lambda}_{1}}{1-q}, a_{i} \rightarrow a \in\left[\frac{d \hat{\lambda}_{1}}{1-q}, A_{0}\right), k_{1, i} \rightarrow \infty, \operatorname{Re} \eta_{i} \leq 0$, and smooth $\left(\omega_{i}, \chi_{i}\right)$ with $\left\|\omega_{i}\right\|_{2}^{2}+\left\|\chi_{i}\right\|_{2}^{2}=1$ such that

$$
\begin{cases}d \Delta \omega_{i}+a(1-q) \omega_{i} h_{1}\left(m_{i} \hat{u}_{i}, v_{i}\right) & \\ -a(1-q) \hat{u}_{i} f_{1}^{\prime}\left(z-m_{i} \hat{u}_{i}-v_{i}, \hat{u}_{i}\right) \chi_{i}+\eta_{i} \omega_{i}=0, & x \in \Omega, \\ d \Delta \chi_{i}+b h_{2}\left(m_{i} \hat{u}_{i}, v_{i}\right) \chi_{i}-q a m_{i} \hat{u}_{i} f_{1}^{\prime}\left(z-m_{i} \hat{u}_{i}-v_{i}, \hat{u}_{i}\right) \chi_{i} & \\ -b m_{i} v_{i} g_{1}^{\prime}\left(z-m_{i} \hat{u}_{i}-v_{i}, v_{i}\right) \omega_{i}+a q m_{i} h_{1}\left(m_{i} \hat{u}_{i}, v_{i}\right) \omega_{i}+\eta_{i} \chi_{i}=0, & x \in \Omega, \\ \frac{\partial \omega_{i}}{\partial n}+r \omega_{i}=0, \quad \frac{\partial \chi_{i}}{\partial n}+r \chi_{i}=0, & x \in \partial \Omega,\end{cases}
$$

where

$$
\begin{aligned}
& h_{1}\left(m_{i} \hat{u}_{i}, v_{i}\right)=f\left(z-m_{i} \hat{u}_{i}-v_{i}, \hat{u}_{i}\right)-m_{i} \hat{u}_{i} f_{1}^{\prime}\left(z-m_{i} \hat{u}_{i}-v_{i}, \hat{u}_{i}\right)+\hat{u}_{i} f_{2}^{\prime}\left(z-m_{i} \hat{u}_{i}-v_{i}, \hat{u}_{i}\right), \\
& h_{2}\left(m_{i} \hat{u}_{i}, v_{i}\right)=g\left(z-m_{i} \hat{u}_{i}-v_{i}, v_{i}\right)-v_{i} g_{1}^{\prime}\left(z-m_{i} \hat{u}_{i}-v_{i}, v_{i}\right)+v_{i} g_{2}^{\prime}\left(z-m_{i} \hat{u}_{i}-v_{i}, v_{i}\right),
\end{aligned}
$$

with a positive solution $\left(\hat{u}_{i}, v_{i}\right)$ of $(22)$ and $(a, m)=\left(a_{i}, \frac{1}{k_{1, i}}\right)$. Multiplying by $\bar{w}_{i}$ and $\hat{\chi}_{i}$ two equations of (23) and integrating over $\Omega$, respectively, it follows from Green's formula that

$$
\begin{aligned}
d \int_{\Omega}\left|\nabla \omega_{i}\right|^{2}= & -r d \int_{\partial \Omega}\left|\omega_{i}\right|^{2}+\int_{\Omega} a(1-q) \omega_{i} h_{1}\left(m_{i} \hat{u}_{i}, v_{i}\right)\left|\omega_{i}\right|^{2} \\
& -\int_{\Omega} a(1-q) \hat{u}_{i} f_{1}^{\prime}\left(z-m_{i} \hat{u}_{i}-v_{i}, \hat{u}_{i}\right) \bar{\omega}_{i} \chi_{i}+\int_{\Omega} \eta_{i}\left|\omega_{i}\right|^{2},
\end{aligned}
$$




$$
\begin{aligned}
d \int_{\Omega}\left|\nabla \chi_{i}\right|^{2}= & -r d \int_{\partial \Omega}\left|\chi_{i}\right|^{2}+\int_{\Omega}\left[b h_{2}\left(m_{i} \hat{u}_{i}, v_{i}\right)-q a m_{i} \hat{u}_{i} f_{1}^{\prime}\left(z-m_{i} \hat{u}_{i}-v_{i}, \hat{u}_{i}\right)\right]\left|\chi_{i}\right|^{2} \\
& -\int_{\Omega}\left[b v_{i} g_{1}^{\prime}\left(z-m_{i} \hat{u}_{i}-v_{i}, v_{i}\right)-a q h_{1}\left(m_{i} \hat{u}_{i}, v_{i}\right)\right] m_{i} \omega_{i} \bar{\chi}_{i}+\int_{\Omega} \eta_{i}\left|\chi_{i}\right|^{2} .
\end{aligned}
$$

Adding these two identities, we get

$$
\begin{aligned}
\eta_{i}= & d \int_{\Omega}\left|\nabla \omega_{i}\right|^{2}+d r \int_{\partial \Omega}\left|\omega_{i}\right|^{2}-\int_{\Omega} a(1-q) h_{1}\left(m_{i} \hat{u}_{i}, v_{i}\right)\left|\omega_{i}\right|^{2} \\
& +\int_{\Omega} a(1-q) \hat{u}_{i} f_{1}^{\prime}\left(z-m_{i} \hat{u}_{i}-v_{i}, \hat{u}_{i}\right) \bar{\omega}_{i} \chi_{i}+d \int_{\Omega}\left|\nabla \chi_{i}\right|^{2}+d r \int_{\partial \Omega}\left|\chi_{i}\right|^{2} \\
& -\int_{\Omega}\left[b h_{2}\left(m_{i} \hat{u}_{i}, v_{i}\right)-q a m_{i} \hat{u}_{i} f_{1}^{\prime}\left(z-m_{i} \hat{u}_{i}-v_{i}, \hat{u}_{i}\right)\right]\left|\chi_{i}\right|^{2} \\
& +\int_{\Omega}\left[b v_{i} g_{1}^{\prime}\left(z-m_{i} \hat{u}_{i}-v_{i}, v_{i}\right)-a q h_{1}\left(m_{i} \hat{u}_{i}, v_{i}\right)\right] m_{i} \omega_{i} \bar{\chi}_{i} .
\end{aligned}
$$

Thanks to the boundedness of $\hat{u}_{i}, v_{i}, a_{i}, m_{i}$, we can easily get that the imaginary part of the right-hand side of this identity is bounded, and thus $\operatorname{Im} \eta_{i}$ is bounded. On the other hand, it is easy to see that $\operatorname{Re} \eta_{i}$ is also bounded. Hence $\eta_{i}$ is bounded with $\operatorname{Re} \eta_{i} \leq 0$. Applying $L^{p}$ estimates, we know that $\left\|\omega_{i}\right\|_{W^{2,2}}$ and $\left\|\chi_{i}\right\|_{W^{2,2}}$ are bounded. We may suppose that $\omega_{i} \stackrel{H_{0}^{1}}{\longrightarrow} \omega$ and $\chi_{i} \stackrel{H_{0}^{1}}{\longrightarrow} \chi$. Since $m_{i} \hat{u}_{i} \rightarrow 0, \hat{u}_{i} \rightarrow w_{0}$, and $v_{i} \rightarrow \theta$ as $i \rightarrow \infty$, taking the limits of two sides of (23), we know that $\omega$ and $\chi$ weakly satisfy the following equations:

$$
\begin{cases}d \Delta \omega+a(1-q) \omega H_{1}-a(1-q) w_{0} f_{1}^{\prime}\left(z-\theta, w_{0}\right) \chi+\eta \omega=0, & x \in \Omega, \\ d \Delta \chi+b \chi H_{2}+\eta \chi=0, & x \in \Omega, \\ \frac{\partial \omega}{\partial n}+r \omega=0, \quad \frac{\partial \chi}{\partial n}+r \chi=0, & x \in \partial \Omega,\end{cases}
$$

where

$$
\begin{aligned}
& H_{1}=f\left(z-\theta, w_{0}\right)+w_{0} f_{2}^{\prime}\left(z-\theta, w_{0}\right), \\
& H_{2}=g(z-\theta, \theta)-\theta g_{1}^{\prime}(z-\theta, \theta)+\theta g_{2}^{\prime}(z-\theta, \theta) .
\end{aligned}
$$

If $\chi \equiv 0$, then $\omega$ satisfies

$$
\begin{cases}d \Delta w+a(1-q) w\left[f\left(z-\theta, w_{0}\right)+w_{0} f_{2}^{\prime}\left(z-\theta, w_{0}\right)\right]+\eta w=0, & x \in \Omega, \\ \frac{\partial w}{\partial n}+r w=0, & x \in \partial \Omega .\end{cases}
$$

This system implies that $\eta$ is real and $\eta>0$, which is a contradiction to $\operatorname{Re} \eta_{i} \leq 0$.

If $\chi \not \equiv 0$, then thanks to $g_{1}^{\prime}(z-\theta, \theta)>0, g_{2}^{\prime}(z-\theta, \theta)<0$, and $\eta \leq 0$, we have

$$
d \Delta \chi+b \chi\left[g(z-\theta, \theta)-\theta g_{1}^{\prime}(z-\theta, \theta)+\theta g_{2}^{\prime}(z-\theta, \theta)\right]+\eta \chi=0, \quad x \in \Omega .
$$

From Remark 2.1 we know that all eigenvalues of $L_{(b, d)}$ are strictly negative, and then $\eta>0$, which contradicts the assumption.

We further prove the existence of a positive solution of (3) with $b>d \mu_{1}$. Because all positive solutions of (3) are nondegenerate, by a simple compactness argument we get 
that there are at most finitely many positive solutions. For some positive solution $(u, v)$, it is easy to see that index $\cos _{W}(F,(u, v))=1$ by the nondegeneracy and stability of $(u, v)$ and Proposition 2.1 in [26]. For $a \in\left[\frac{\hat{\lambda}_{1} d}{1-q}+\varepsilon, A\right]$, by Theorem 2.2, (3) has at least one positive solution. Let the finitely many positive solutions of (3) be $\left\{\left(u_{i}, v_{i}\right): 1 \leq i \leq l\right\}, l \geq 1$. Applying Lemmas 2.3-2.4 and the additivity of the fixed point index, we obtain

$$
1=\operatorname{index}_{W}\left(F, D^{\prime}\right)=0+\sum_{i=1}^{l} \operatorname{index}_{W}\left(F,\left(u_{i}, v_{i}\right)\right)=l,
$$

which proves the uniqueness of positive solutions of (3).

Finally, we investigate the case $a=\frac{\hat{\lambda}_{1} d}{1-q}$. By the regular bifurcation theory, taking $a$ as a bifurcation parameter, we can construct a positive solution branch from the semitrivial nonnegative solution branch $\left\{\left(\frac{\hat{\lambda}_{1} d}{1-q}, 0, \theta\right), a \in R^{+}\right\}$. The positive bifurcation solution cave (see Theorem 2.4.1 in [26]) is

$$
\Gamma_{m}=\left\{(a(s), u(s), v(s))=\left(a(s), s\left(\hat{\phi}_{1}+\phi(s), \theta-s\left(\chi_{1}+\psi(s)\right)\right)\right), 0<s \ll 1\right\},
$$

where $a(0)=\frac{\hat{\lambda}_{1} d}{1-q}, \phi(0)=0, \psi(0)=0$, and

$$
\chi_{1}=-L_{b}^{-1}\left[b m \theta g_{1}^{\prime}(z-\theta, \theta)-\operatorname{aqmf}(z-\theta, 0) \hat{\phi}_{1}\right] .
$$

Substituting the positive solution into the first equation of (3), dividing by $s$, and differentiating on $s$, we can get the derivative on both sides of the equation at $s=0$, and hence

$$
a^{\prime}(0)(1-q) \int_{\Omega} f(z-\theta, 0) \hat{\phi}_{1}^{2} d x=d \hat{\lambda}_{1} \int_{\Omega}\left[\left(m \hat{\phi}_{1}-\chi_{1}\right) f_{1}^{\prime}(z-\theta, 0)-f_{2}^{\prime}(z-\theta, 0) \hat{\phi}_{1}\right] \hat{\phi}_{1}^{2} d x .
$$

Letting $k_{1} \rightarrow \infty$, that is, $m \rightarrow 0$, we get

$$
a^{\prime}(0)=-\frac{d \hat{\lambda}_{1}}{1-q} \frac{\int_{\Omega}\left(f_{2}^{\prime}(z-\theta, 0) \hat{\phi}_{1}\right) \hat{\phi}_{1}^{2} d x}{\int_{\Omega} f(z-\theta, 0) \hat{\phi}_{1}^{2} d x}>0 .
$$

Since $\chi_{1} \rightarrow 0$ and $f_{2}^{\prime}(z-\theta, 0)<0$, it is easy to see that the positive solution bifurcation branch lies in the right. According to Theorem 2.4.3 in [26] and [5], we can prove that $\Gamma_{m}$ can be extended to the global bifurcation solution along the parameter $a>\frac{d \hat{\lambda}_{1}}{1-q}$, and it tends to infinity. Hence, as $a \in\left(\frac{d \hat{\lambda}_{1}}{1-q}+\varepsilon, A\right]$ and $k_{1} \rightarrow \infty$, the positive solution of (3) is uniquely determined on $\Gamma_{m}$, and no positive solution curve can cover $a=\frac{d \hat{\lambda}_{1}}{1-q}$. Thus there is no positive solution of (3) when $a=\frac{d \hat{\lambda}_{1}}{1-q}$. The proof of Theorem 4.2 is complete.

\section{Asymptotic behavior of solutions}

The goal of this section is to present some asymptotic behavior of solutions of (1) depending on the change of time by comparison principle and persistence theorem.

Lemma 5.1 System (1) has nonnegative bounded solutions $S(t, x), u(t, x), v(t, x)$, and for some $\alpha$,

$$
\|S(t, \cdot)+u(t, \cdot)+v(t, \cdot)-z\|_{\infty}=O\left(e^{-\alpha t}\right) \quad(t \rightarrow \infty)
$$

where $z=z(x)=S^{0}\left(\frac{1+r}{r}-x\right)$. 
Proof By Theorem 14.2 in [27] we can get the local existence of solutions. The nonnegativity of solutions can be proved by the comparison principle of parabolic equations.

Let $w(x, t)=S(t, x)+u(t, x)+v(t, x)-z(x)$. Since $z(x)$ satisfies

$$
\begin{cases}d \Delta z=0, & x \in \Omega, \\ \frac{\partial z}{\partial n}+r z=S^{0}, & x \in \partial \Omega,\end{cases}
$$

it follows that $w(t, x)$ satisfies

$$
\begin{cases}w_{t}=d \Delta w, & x \in \Omega, \\ \frac{\partial w}{\partial n}+r w=0, & x \in \partial \Omega, \\ w(0, x)=S(0, x)+u(0, x)+v(0, x)-z(x), & x \in \Omega,\end{cases}
$$

where $w(t, x)=\phi(x) Y(t, x) e^{-\alpha t}(\alpha>0)$ with the principal eigenfunction $\phi(x)$ of the following problem:

$$
\begin{cases}d \Delta \phi+\lambda \phi=0, & x \in \Omega \\ \frac{\partial \phi}{\partial n}+r \phi=0, & x \in \partial \Omega .\end{cases}
$$

If $\eta_{0}$ is the principal eigenvalue, then $\phi(x)>0(x \in \Omega)$.

Substituting $w(t, x)$ into equation (25), we get

$$
\begin{cases}d \Delta Y-Y_{t}+2 d \frac{\nabla \phi}{\phi} \nabla Y+\left(-\eta_{0}+\alpha\right) Y=0, & x \in \Omega, \\ \frac{\partial Y}{\partial n}=0, & x \in \partial \Omega .\end{cases}
$$

Suppose $\alpha$ satisfies $0<\alpha<\eta_{0}$. Applying the maximum principle, we get that the maximum value of $Y(T, x)$ cannot be taken on the interior and the border of the region, and hence $Y(t, x) \leq \max _{z \in \bar{\Omega}} Y(0, x)$. Similarly, for (26), replacing $Y$ by $-Y$, we have $Y(t, x) \geq$ $-\min _{z \in \bar{\Omega}} Y(0, x)$. Thus, there exists $\hat{C}>0$ such that $|Y(t, x)| \leq \hat{C}$, so that $Y(t, x)$ is bounded, and the proof is complete.

From Lemma 5.1 and from $z(x)=S(t, x)+u(t, x)+v(t, x)$ we get that $u$ and $v$ satisfy the following problem:

$$
\begin{cases}u_{t}=d \Delta u+(1-q) \operatorname{auf}(z-u-v, u), & x \in \Omega, t>0, \\ v_{t}=d \Delta v+b v g(z-u-v, v)+q a u f(z-u-v, u), & x \in \Omega, t>0, \\ \frac{\partial u}{\partial n}+r u=0, \quad \frac{\partial v}{\partial n}+r v=0, & x \in \partial \Omega, t>0, \\ u(0, x)=u_{0}(x) \geq 0, \not \equiv 0, & x \in \Omega, \\ v(0, x)=v_{0}(x) \geq 0, \not \equiv 0, & x \in \Omega,\end{cases}
$$

where $z(x)=S(x)+u(x)+v(x)$, and $(S(x), u(x), v(x))$ is the equilibrium solution of (1). By the skills in $[27,28]$ we can deduce that $(27)$ has a solution $(u(x), v(x))$ in a small neighborhood. Observing that $u_{0}(x) \geq 0, \not \equiv 0, v_{0}(x) \geq 0, \not \equiv 0$, by the comparison principle of parabolic equations we get $u(t, x)>0, v(t, x)>0, x \in \Omega, t>0$. 
Obviously, $v(t, x) \equiv 0$ implies $u(t, x) \equiv 0$. Therefore, system (27) has no single species on $u$. If the initial value of (27) $u_{0}(x) \equiv 0$, then by the maximum principle we know $u(T, x) \equiv 0$. Then $v$ satisfies the system

$$
\begin{cases}v_{t}=d \Delta v+b v g(z-v, v), & x \in \Omega, t>0 \\ \frac{\partial v}{\partial n}+r v=0, & x \in \partial \Omega, t>0 \\ v(0, x)=v_{0}(x) \geq 0, \not \equiv 0, & x \in \Omega\end{cases}
$$

By Theorems 3.1-3.2 and the partial lemmas of [12] there are some conclusions about the persistence and extinction of the single species $v$.

Theorem 5.1 Suppose $v(t, x)$ is a solution of (28). If $b<\mu_{1} d$, then $\lim _{t \rightarrow \infty} v(t, x)=0$; if $b>\mu_{1} d$, then $\lim _{t \rightarrow \infty} \sup \|v(t, \cdot)\|_{\infty}>0$.

Theorem 5.2 Suppose $v(t, x)$ is a solution of (28). If $b>\mu_{1} d$, then there exists a unique positive solution $\theta$ of the equilibrium equation on (28), and $\lim _{t \rightarrow \infty} v(t, x)=\theta$.

Based on the single species conclusion, we investigate the asymptotic behavior of the solution of system (1). Similarly to Theorem 5.2 , we obtain the following lemma.

Lemma 5.2 Suppose $u(t, x)$ is a solution of the following equation:

$$
\begin{cases}u_{t}=d \Delta u+a(1-q) u f(z-u, u), & x \in \Omega, t>0, \\ \frac{\partial u}{\partial n}+r u=0, & x \in \partial \Omega, t>0 \\ u(0, x)=u_{0}(x) \geq 0, \not \equiv 0, & x \in \Omega .\end{cases}
$$

Then, as $a<\frac{\lambda_{1} d}{1-q}, \lim _{t \rightarrow \infty} u(t, x)=0$, and as $a>\frac{\lambda_{1} d}{1-q}, \lim _{t \rightarrow \infty} u(t, x)=\Theta$.

Consider the following problem:

$$
\begin{cases}v_{t}=d \Delta v+b v g(z-v, v)+a q \zeta z f(z-v, 0), & x \in \Omega, t>0, \\ \frac{\partial v}{\partial n}+r v=0, & x \in \partial \Omega, t>0, \\ v(0, x)=v_{0}(x) \geq 0, \not \equiv 0, & x \in \Omega .\end{cases}
$$

\section{Lemma 5.3}

(i) If $b>\mu_{1} d$, then there exists a unique solution $\theta^{5}$ for the equilibrium equation of (29);

(ii) Suppose that $v^{\varsigma}(t, x)$ is a positive solution of (29) and $\theta$ is a unique positive solution of $(7)$.

If $b<\mu_{1} d$, then $\lim _{t \rightarrow \infty, \varsigma \rightarrow 0} \nu^{\varsigma}(t, x)=0$;

If $b>\mu_{1} d$, then $\lim _{t \rightarrow \infty, \varsigma \rightarrow 0} \nu^{\varsigma}(t, x)=\theta$.

Proof (i) If $b>\mu_{1} d$, by a method similar to the proof on Lemma 2.2 of [7] it is easy to get the existence and uniqueness of $\theta^{5}$, and $0<\theta^{5}<z$. 
(ii) If $b<\mu_{1} d$, then by Lemma 5.1, for any given $\varepsilon>0$, there exists $t_{0}>0$ such that $S(t, x) \leq$ $z(x)+\varepsilon$. For all $t \geq t_{0}, x \in \bar{\Omega}$, suppose that $V^{\varsigma}(t, x)$ is a solution of the following problem:

$$
\begin{cases}V_{t}^{\varsigma}=d \Delta V^{\varsigma}+b V^{\varsigma} g(z+\varepsilon, 0)+a q \varsigma z f(z+\varepsilon, 0), & x \in \Omega, t>0, \\ \frac{\partial V^{\varsigma}}{\partial n}+r V^{\varsigma}=0, & x \in \partial \Omega, t>0, \\ V^{\varsigma}\left(t_{0}, x\right)>v^{\varsigma}\left(t_{0}, x\right), & x \in \Omega .\end{cases}
$$

Next, we prove that $V^{\varsigma}(t, x)>v^{\varsigma}(t, x), t \geq t_{0}, x \in \bar{\Omega}$. Let $w(t, x)=v^{\varsigma}(t, x)-V^{\varsigma}(t, x)$. Then

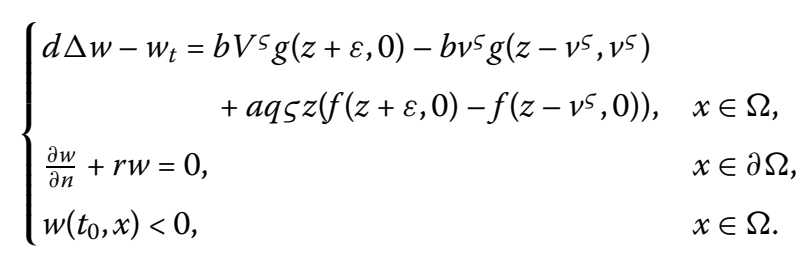

Then we only need to prove that $w(t, x)<0, t \geq t_{0}, x \in \bar{\Omega}$. Assume that the conclusion does not hold. Then, let $t_{1}$ be the first moment such that there exists $x_{1}$ such that $w\left(t_{1}, x_{1}\right)=0$, and thus, for $x \in \Omega$ and $t_{0}<t \leq t_{1}$, we have

$$
d \Delta w-w_{t} \geq 0
$$

By the maximum principle the nonnegative maximum of $w$ lies on $C_{1}=\left\{t_{0} \leq t \leq t_{1}, x \in\right.$ $\partial \Omega\}$ or $C_{2}=\left\{t=t_{0}, x \in \Omega\right\}$. Because of the initial condition $w\left(t_{0}, x\right)<0$, we remove the case of $C_{2}$. The case of $C_{1}$ implies $\frac{\partial w}{\partial n}>0$. However, $\frac{\partial w}{\partial n}=-r w \leq 0, x \in \partial \Omega$, a contradiction.

Let $V^{\varsigma}=Y^{\varsigma}(t, x) \psi e^{-\alpha\left(t-t_{0}\right)}$, where $\psi$ is the principal eigenfunction of the following problem:

$$
\begin{cases}d \Delta \psi+\mu g(z, 0) \psi=0, & x \in \Omega \\ \frac{\partial \psi}{\partial n}+r \psi=0, & x \in \partial \Omega\end{cases}
$$

If $\mu_{1}$ is the corresponding principal eigenvalue, then

$$
\begin{cases}d \Delta Y^{\varsigma}-Y_{t}^{\varsigma}+2 d \frac{\nabla \psi}{\psi} \nabla Y^{\varsigma}+\frac{1}{\psi} C(x) Y^{\varsigma}+a q \varsigma z f(z+\varepsilon, 0) e^{\alpha\left(t-t_{0}\right)}=0, & x \in \Omega, \\ \frac{\partial Y^{\varsigma}}{\partial n}=0, & x \in \partial \Omega,\end{cases}
$$

where

$$
\begin{aligned}
C(x) & =d \Delta \psi+\alpha \psi+b g(z+\varepsilon, 0) \psi=-d \mu_{1} g(z, 0) \psi+\alpha \psi+b g(z+\varepsilon, 0) \psi \\
& =\alpha \psi+(b g(z+\varepsilon, 0) \psi-b g(z, 0) \psi)+\left(b g(z, 0) \psi-d \mu_{1} g(z, 0) \psi\right) .
\end{aligned}
$$

Because $b<\mu_{1} d$ and $\varepsilon, \alpha$ are sufficiently small, $C(x)$ is less than zero, and if $\varsigma$ is small enough, then

$$
d \Delta Y^{\varsigma}-Y_{t}^{\varsigma}+2 d \frac{\nabla \psi}{\psi} \nabla Y^{\varsigma}>0
$$


By the maximum principle we have $Y^{\varsigma}(t, x) \leq Y^{\varsigma}\left(t_{0}, x\right)=\sup _{x \in \bar{\Omega}}\left(\frac{V \zeta\left(t_{0}, x\right)}{\psi}\right)$, and it follows that there exists $K>0$ such that $v^{\varsigma}(t, x) \leq V^{\zeta}(t, x) \leq K e^{-\alpha\left(t-t_{0}\right)}$.

If $b>\mu_{1} d$, by Lemma 3.3 in [12] we can obtain that $\lim _{t \rightarrow \infty} \sup \left\|v^{\varsigma}(t, \cdot)\right\|_{\infty}>0$. Next, we construct th Lyapunov function

$$
L(\psi)=\int_{\Omega}\left[\frac{d}{2}(\nabla \psi)^{2}-\hbar(x, \psi(x))\right] d x+B(\psi)
$$

where

$$
\begin{aligned}
& \hbar(x, v)=\int_{\Omega} f(x, s) d s, \quad f(x, v)=b g(z-v, v) v+\operatorname{aq\varsigma } z f(z-v, 0), \\
& B(\psi)=\int_{\partial \Omega} \frac{d r}{2} \psi^{2} d s .
\end{aligned}
$$

Then

$$
\frac{\partial L\left(v^{\varsigma}(t, x)\right)}{\partial t}=-\int_{\Omega}\left(v_{t}^{\varsigma}\right)^{2} d x \leq 0
$$

Since $v^{\varsigma}(t, x)$ is close to the invariant set which satisfies $\frac{\partial L(\nu \varsigma(t, x))}{\partial t}=0$, observe that $\frac{\partial L\left(v^{\varsigma}(t, x)\right)}{\partial t}=0$ if and only if $v^{\varsigma}(t, x)=0$, that is, the set of equilibrium states of equation (28).

Combining Lemma 5.2, equation (29), and the uniqueness of the positive equilibrium solution $\theta^{\varsigma}$, we obtain $\lim _{t \rightarrow \infty} v^{5}(t, x)=\theta^{\varsigma}$.

In the remaining part, we prove that $\lim _{5 \rightarrow 0} \theta^{\varsigma}=\theta$. Since $0<\theta^{\varsigma}<z$, there exists a constant $K>0$, independent of $\zeta$, such that, for any $p \geq 1$,

$$
\left\|\theta^{\varsigma}\right\|_{2, p} \leq K^{\prime}\left(\left\|\theta^{\varsigma}\right\|_{p}+\left\|b g\left(z-\theta^{\varsigma}, \theta^{\varsigma}\right) \theta^{\varsigma}+\operatorname{aq} \varsigma z f\left(z-\theta^{\varsigma}, 0\right)\right\|_{p}\right) \leq K .
$$

By the Sobolev imbedding theorem we have $\theta^{\varsigma} \rightarrow \theta^{0}, \varsigma \rightarrow 0$, and $0<\theta^{0}<z$, so that $\theta^{0}$ weakly satisfies the following equation:

$$
\begin{cases}d \Delta \theta^{0}+b \theta^{0} g\left(z-\theta^{0}, \theta^{0}\right)=0, & x \in \Omega, \\ \frac{\partial \theta^{0}}{\partial n}+r \theta^{0}=0, & x \in \partial \Omega .\end{cases}
$$

By the regularization theory of elliptic equations we get $\theta^{0} \in C^{2}$. Hence, by the uniqueness of $\theta$ we get that $\theta^{0}=\theta$, and the proof is complete.

Similarly to Lemma 5.3, we can establish the following lemmas.

Lemma 5.4 Suppose $v^{\varsigma}(t, x)$ is a solution of the following problem:

$$
\begin{cases}v_{t}=d \Delta v+b v g(z-\varsigma z-v, v), & x \in \Omega, t>0, \\ \frac{\partial v}{\partial n}+r v=0, & x \in \partial \Omega, t>0, \\ v(0, x)=v_{0}(x) \geq 0, & x \in \Omega .\end{cases}
$$

If $b>\mu_{1} d$, then $\lim _{t \rightarrow \infty, \zeta \rightarrow 0} \nu^{\varsigma}(t, x)=\theta$. 
Lemma 5.5 Suppose that $a<\frac{\lambda_{1} d}{1-q}$ and that $u(t, x), v(t, x)$ are solutions of (27).

(1) If $b<\mu_{1} d$, then $\lim _{t \rightarrow \infty} u(t, x)=0$ and $\lim _{t \rightarrow \infty} v(t, x)=0$;

(2) If $b>\mu_{1} d$, then $\lim _{t \rightarrow \infty} u(t, x)=0$ and $\lim _{t \rightarrow \infty} v(t, x)=\theta$.

Proof Since $a<\frac{\lambda_{1} d}{1-q}$, it is easy to see that

$$
u_{t}=d \Delta u+a(1-q) f(z-u-v, u) u<d \Delta u+a(1-q) f(z-u, u) u
$$

Suppose $U(t, x)$ is a solution of the following problem:

$$
\begin{cases}U_{t}=d \Delta U+a(1-q) f_{1}(z-U, U) U, & x \in \Omega, t>0, \\ \frac{\partial U}{\partial n}+r U=0, & x \in \partial \Omega, t>0, \\ U\left(t_{0}, x\right)>u\left(t_{0}, x\right), & x \in \Omega .\end{cases}
$$

By the comparison principle we get $0<u<U$. By Lemma 5.4, if $a<\frac{\lambda_{1} d}{1-q}$, then $\lim _{t \rightarrow \infty} U(t$, $x)=0$. Thus $\lim _{t \rightarrow \infty} u(t, x)=0$.

Thus, $-\varsigma z<u<\varsigma z$, where $\varsigma$ is a sufficiently small number, and it follows that

$$
\begin{aligned}
v_{t} & =d \Delta v+b g(z-u-v, v) v+a q f(z-u-v, u) u \\
& \leq d \Delta v+b g(z-v, v) v+a q \varsigma z f(z-v, 0)
\end{aligned}
$$

and

$$
v_{t}=d \Delta v+b g(z-u-v, v) v+a q f(z-u-v, u) u \geq d \Delta v+b g(z-\varsigma z-v, v) v .
$$

Suppose $\bar{v}(t, x), \underline{v}(t, x)$ are two solutions of the following two equations, respectively:

$$
\begin{aligned}
& \begin{cases}\bar{v}_{t}=d \Delta \bar{v}+b g(z-\bar{v}, \bar{v}) \bar{v}+a q \varsigma z f(z-\bar{v}, 0), & x \in \Omega, t>0, \\
\frac{\partial \bar{v}}{\partial n}+r \bar{v}=0, & x \in \partial \Omega, t>0, \\
\bar{v}\left(t_{0}, x\right)>v\left(t_{0}, x\right), & x \in \Omega,\end{cases} \\
& \begin{cases}\underline{v}_{t}=d \Delta \underline{v}+b g(z-\varsigma z-\underline{v}, \underline{v}) \underline{v}, & x \in \Omega, t>0, \\
\frac{\partial \underline{v}}{\partial n}+r \underline{v}=0, & x \in \partial \Omega, t>0, \\
\underline{v}\left(t_{0}, x\right)<v\left(t_{0}, x\right), & x \in \Omega .\end{cases}
\end{aligned}
$$

For (30) and (31), by the comparison principle we obtain $0<v(t, x)<\bar{v}(t, x), \underline{v}(t, x)<v(t, x)<$ $\bar{v}(t, x)$. By Lemmas 5.3-5.4 we can deduce the conclusion as follows. If $b<\mu_{1} d$, then $\lim _{t \rightarrow \infty, 5 \rightarrow 0} \bar{v}(t, x)=0$, and therefore $\lim _{t \rightarrow \infty} v(t, x)=0$. If $b>\mu_{1} d$, then $\lim _{t \rightarrow \infty, 5 \rightarrow 0} \bar{v}(t, x)=$ $\theta$ and $\lim _{t \rightarrow \infty, 5 \rightarrow 0} \underline{v}(t, x)=\theta$, and thus $\lim _{t \rightarrow \infty} v(t, x)=\theta$. Thus, the lemma is proved.

Based on Lemmas 5.2-5.5, we can obtain the asymptotic behavior of solutions of system (1) as follows.

Theorem 5.3 Suppose that $a<\frac{\lambda_{1} d}{1-q}$ and that $S(t, x), u(t, x), v(t, x)$ is the positive solution of (1). Then the following results hold: 
(i) If $b<\mu_{1} d$, then $\lim _{t \rightarrow \infty}(S(t, x), u(t, x), v(t, x))=(z(x), 0,0)$;

(ii) If $b>\mu_{1} d$, then $\lim _{t \rightarrow \infty}(S(t, x), u(t, x), v(t, x))=(z(x)-\theta, 0, \theta)$.

Finally, we establish the uniform persistence of system (27), which suggests that two species can coexist.

Theorem 5.4 Suppose $a>\frac{\lambda_{1} d}{1-q}$ and $b<d \mu_{1}$. Then there exists $\tilde{\varrho}>0$, and for any solution of (27), there exists $\bar{t}_{0}>0$ (depending on the initial conditions) such that $\min _{x \in \bar{\Omega}} u(t, x)>\tilde{\varrho}$ as $t>\bar{t}_{0}$. Hence, the semidynamical system produced by (27) is strongly consistent continuous.

Theorem 5.5 Suppose $a>\frac{\hat{\lambda}_{1} d}{1-q}$ and $b>d \mu_{1}$. Then there exists $\varrho>0$, and for any solution of (27), there exists $t_{0}>0$ (depending on the initial conditions) such that $\min _{x \in \bar{\Omega}} u(t, x)>\varrho$ as $t>t_{0}$. Hence, the semidynamical system produced by (27) is strongly consistent continuous.

Proof Applying the persistence theorem in [29, 30] to prove Theorems 5.4-5.5, because the proof process is similar, we only prove Theorem 5.5. Suppose that the state space of semidynamic systems produced by (1) is defined as $Y=C^{+}(\bar{\Omega}) \times C^{+}(\bar{\Omega})=X_{1} \cup X_{2}$, where $X_{1}=\left\{(u, v) \in Y: \exists x_{0} \in \Omega\right.$ s.t. $\left.u\left(x_{0}\right)>0\right\}$ and $X_{2}=\{(u, v) \in Y: u \equiv 0\}$. It is easy to see that $X_{1} \subset Y$ is open invariant set, the equilibrium state $(0,0),(0, \theta) \in X_{2}$, and $X_{2}$ is also an invariant set. By Theorem 5.3 we know that $v(t, x) \rightarrow \theta(t \rightarrow \infty)$ as $b>\mu_{1} d$, so $(0, \theta)$ attracts $(0, v)(v \geq, \not \equiv 0)$. Hence the $\omega$-set of orbitals starting at $X_{2}$ is defined by $\Omega_{2}=\{(0,0),(0, \theta)\}$. Let $M_{1}=\{(0,0)\}$ and $M_{2}=\{(0, \theta)\}$. Then $M=\left\{\left(M_{1}, M_{2}\right)\right\}=\{(0,0),(0, \theta)\}$, Obviously, $M_{1}$ can connect to $M_{2}$, but $M_{2}$ cannot connect to $M_{1}$. So $M$ is an acyclic isolated covering $\Omega_{2}$. Next, we only prove that $M_{i}(i=1,2)$ is weakly exclusive to $X_{1}$ and $M$ is isolated.

Suppose $M_{2}$ is not weakly exclusive to $X_{1}$. Then there exists $\left(u_{0}, v_{0}\right) \in X_{1}$ such that

$$
(u(t, x), v(t, x)) \rightarrow(0, \theta)
$$

uniformly on $\bar{\Omega}$ as $t \rightarrow \infty$. Since $a>\frac{\hat{\lambda}_{1} d}{1-q}$, along with the continuity of the principal eigenvalue, we know that there exists $\epsilon>0$ such that the principal eigenvalue $\hat{\lambda}_{1}^{\epsilon}$ of the following problem satisfies $a>\frac{\hat{\lambda}_{1}^{\epsilon} d}{1-q}$ :

$$
\begin{cases}\Delta \phi+\phi \hat{\lambda}_{1}^{\epsilon} f((1-\epsilon) z-\theta, 0)=0, & x \in \Omega, t>0, \\ \frac{\partial \phi}{\partial n}+r \phi=0, & x \in \partial \Omega, t>0 .\end{cases}
$$

On the other hand, $\lim _{t \rightarrow \infty}(u(t, x), v(t, x))=(0, \theta)$, and thus there exists $t_{0}$ such that $0<$ $v(t, x)<\theta+\epsilon z$ for $x \in \bar{\Omega}$ as $t \geq t_{0}$. Hence

$$
u_{t} \geq d \Delta u+a(1-q) f((1-\epsilon) z-u-\theta, u) u
$$

Applying the comparison principle, we have $u(t, x)>h(t, x)$ as $t \geq t_{0}$, where $h(t, x)$ satisfies

$$
\begin{cases}h_{t}=d \Delta h+a(1-q) h f((1-\epsilon) z-h-\theta, h), & x \in \Omega, t>t_{0}, \\ \frac{\partial h}{\partial n}+r h=0, & x \in \partial \Omega, t>t_{0}, \\ h\left(t_{0}, x\right)=\min \{u(t, x),(1-\epsilon) z\}, & x \in \Omega .\end{cases}
$$


Obviously, if $a>\frac{\hat{\lambda}_{1}^{\epsilon} d}{1-q}$, then (32) has a unique positive equilibrium solution $h^{\epsilon}(x)$, and $\lim _{t \rightarrow \infty} h(t, x)=h^{\epsilon}(x)$. By $L^{p}$ estimate and the Sobolev embedding theorem we get that $h^{\epsilon}(x) \rightarrow h^{0}(x)$ as $\epsilon \rightarrow 0$ on $C^{1}$, where $h^{0}(x)$ is a unique positive solution of the following problem:

$$
d \Delta h^{0}+a(1-q) h^{0} f\left(z-h^{0}-\theta, h^{0}\right)=0, \quad \frac{\partial h^{0}}{\partial n}+r h^{0}=0 .
$$

Then there exists $T>t_{0}>0$ such that $u(t, x)>\frac{1}{2} h^{0}$ as $t \geq T$, a contradiction. Thus, $M_{2}$ is a weak exclusion, and it is an isolated invariant set in $Y$. For the invariant set $M_{1}$, since $a>\frac{\hat{\lambda}_{1} d}{1-q}>\frac{\lambda_{1} d}{1-q}$, we can similarly prove that $M_{1}$ is a weak exclusion, and it is an isolated invariant set in $Y$. By Theorem 4.6 in [30], $u$ is uniformly persistent, and for (27), $v$ is also uniformly persistent. Therefore, the semidynamical system is restricted to $X_{1}$ and has a compact invariant attractor $B$ (see Theorem 3.2 in [29]); $B$ attracts the bounded set of $X_{1}$ the distance of which from $X_{2}$ is greater than zero, Particularly, $B$ attracts the point of $X_{1}$. Because the arbitrary orbital of the $X_{1}$ is uniformly attracted to $B$, we only prove that $\varrho>0$ for all $(u, v) \in B$, according to $\min _{x \in \bar{\Omega}} u(x)>\varrho$. Either the sequence, $\left(u_{i}, v_{i}\right) \in B, x_{i} \in \bar{\Omega}$, such that $u_{i}\left(x_{i}\right) \rightarrow 0$. We suppose that the subsequence $\left(u_{i}, v_{i}\right) \rightarrow(u, v) \in B, x_{i} \rightarrow x \in \bar{\Omega}$, and $u(x)=0$. However, $B$ is invariant, and we have $u>0(x \in \bar{\Omega})$, a contradiction.

\section{Conclusion}

This paper deals with plasmid-bearing and plasmid-free models in the unstirred chemostat with the Beddington-DeAngelis functional response. Applying the fixed point theory, bifurcation theory, and the perturbation technique, we obtained the following result: Firstly, some a priori estimates and a sufficient condition for the existence for positive solutions are established. Secondly, we study the effect of the small parameter $k_{1}$ and sufficiently large $k_{2}$ in Beddington-DeAngelis functional response, and we find that the model has at least two positive solutions (Theorem 3.1). Thirdly, we investigate the case of $k_{1}$. The results show that if $k_{1}$ is sufficiently large, then the positive solution of this model is determined by a limiting equation (Lemma 4.1 and Theorems 4.1-4.2). Finally, in Sect. 5, we present some asymptotic behavior of solutions depending on the change of time by the comparison principle and persistence theorem (Theorems 5.3-5.5).

\footnotetext{
Acknowledgements

This work is supported by Shandong Provincial Natural Science Foundation (No. ZR2015AQ001), National Natural Science Foundation of China (Nos. 11371230, 61102144), Shaanxi Province Department of Education Fund (14JK1353), Project for Higher Educational Science and Technology Program of Shandong Province (No. 13LI05), Research Funds for Joint Innovative Center for Safe and Effective Mining Technology and Equipment of Coal Resources by Shandong Province and SDUST Research Fund (2014TDJH102), The president of Xi'an Technological University Foundation (XAGDJJ1423, 17028).

Competing interests

The authors declare that they have no competing interests.

Authors' contributions

All authors worked together to produce the results and read and approved the final manuscript.

Author details

${ }^{1}$ Department of Mathematics and Physics, Xi'an Technological University, Xi'an, P.R. China. ${ }^{2}$ College of Mathematics, Shaanxi Normal University, Xi'an, P.R. China. ${ }^{3}$ College of Mathematics and Systems Science, Shandong University of Science and Technology, Qingdao, P.R. China. ${ }^{4}$ State Key Laboratory of Mining Disaster Prevention and Control Co-founded by Shandong Province and the Ministry of Science and Technology, Shandong University of Science and Technology, Qingdao, P.R. China.
} 


\section{Publisher's Note}

Springer Nature remains neutral with regard to jurisdictional claims in published maps and institutional affiliations.

\section{Received: 2 November 2017 Accepted: 3 April 2018 Published online: 16 May 2018}

\section{References}

1. Feng, X., Tian, J., Ma, X.: Coexistence of an unstirred chemostat model with B-D functional response by fixed point index theory. J. Inequal. Appl. 2016(1), 294 (2016)

2. Stewart, F.M., Levin, B.R.: Partitioning of resources and the outcome of interspecific competition: a model and some general considerations. Am. Nat. 107(954), 171-198 (1973)

3. Hsu, S.B., Hubbell, S., Waltman, P.: A mathematical theory for single-nutrient competition in continuous cultures of micro-organisms. SIAM J. Appl. Math. 32(2), 366-383 (1977)

4. Smith, H., Waltman, P.: The Theory of the Chemostat. Cambridge University Press, Cambridge (1995)

5. Wu, J.H.: Global bifurcation of coexistence state for the competition model in the chemostat. Nonlinear Anal., Theory Methods Appl. 39(7), 817-835 (2000)

6. Jianhua, W., Wolkowicz, G.S.K.: A system of resource-based growth models with two resources in the unstirred chemostat. J. Differ. Equ. 172(2), 300-332 (2001)

7. Wu, J., Nie, H., Wolkowicz, G.S.K.: A mathematical model of competition for two essential resources in the unstirred chemostat. SIAM J. Appl. Math. 65(1), 209-229 (2004)

8. Nie, H., Wu, J.: A system of reaction-diffusion equations in the unstirred chemostat with an inhibitor. Int. J. Bifurc. Chaos 16(4), 989-1009 (2006)

9. Nie, H., Wu, J.: Asymptotic behavior of an unstirred chemostat model with internal inhibitor. J. Math. Anal. Appl. 334(2), 889-908 (2007)

10. Wu, J., Nie, H., Wolkowicz, G.S.K.: The effect of inhibitor on the plasmid-bearing and plasmid-free model in the unstirred chemostat. SIAM J. Math. Anal. 38(6), 1860-1885 (2007)

11. Beddington, J.R.: Mutual interference between parasites or predators and its effect on searching efficiency. J. Anim. Ecol. 44(1), 331-340 (1975)

12. Hsu, S.-B., Waltman, P.: On a system of reaction-diffusion equations arising from competition in an unstirred chemostat. SIAM J. Appl. Math. 53(4), 1026-1044 (1993)

13. Stephanopoulos, G., Lapudis, G.R.: Chemostat dynamics of plasmid-bearing, plasmid-free mixed recombinant cultures. Chem. Eng. Sci. 43(1), 49-57 (1988)

14. Hsu, S.-B., Waltman, P.: A model of the effect of anti-competitor toxins on plasmid-bearing, plasmid-free competition. Taiwan. J. Math. 6(1), 135-155 (2002)

15. Hsu, S.-B., Waltman, P., Wolkowicz, G.S.K.: Global analysis of a model of plasmid-bearing, plasmid-free competition in a chemostat. J. Math. Biol. 32(7), 731-742 (1994)

16. Nie, $\mathrm{H} ., \mathrm{Wu}, \mathrm{J}$ : Coexistence of an unstirred chemostat model with Beddington-DeAngelis functional response and inhibitor. Nonlinear Anal., Real World Appl. 11(5), 3639-3652 (2010)

17. Wang, Y., Wu, J., Guo, G.: Coexistence and stability of an unstirred chemostat model with Beddington-DeAngelis function. Comput. Math. Appl. 60(8), 2497-2507 (2010)

18. Meng, X., Gao, Q., Li, Z.: The effects of delayed growth response on the dynamic behaviors of the Monod type chemostat model with impulsive input nutrient concentration. Nonlinear Anal., Real World Appl. 11(5), 4476-4486 (2010)

19. Meng, X., Wang, L., Zhang, T.: Global dynamics analysis of a nonlinear impulsive stochastic chemostat system in a polluted environment. J. Appl. Anal. Comput. 6, 86-875 (2016)

20. Zhang, T., Ma, W., Meng, X.: Global dynamics of a delayed chemostat model with harvest by impulsive flocculant input. Adv. Differ. Equ. 2017(1), 115 (2017)

21. Gao, J., Shen, B., Feng, E., Xiu, Z.: Modelling and optimal control for an impulsive dynamical system in microbial fed-batch culture. Comput. Appl. Math. 32(2), 275-290 (2013)

22. LV, X., Wang, L., Meng, X.: Global analysis of a new nonlinear stochastic differential competition system with impulsive effect. Adv. Differ. Equ. 2017(1), 297 (2017)

23. Zhang, T., Zhang, T., Meng, X.: Stability analysis of a chemostat model with maintenance energy. Appl. Math. Lett. 68 , 1-7 (2017)

24. DeAngelis, D.L., Goldstein, R.A., O'Neill, R.V.: A model for tropic interaction. Ecology 56(4), 881-892 (1975)

25. Dancer, E.N.: On the indices of fixed points of mappings in cones and applications. J. Math. Anal. Appl. 91(1), 131-151 (1983)

26. Feng, X: The equilibrium and the long time behavior of two kinds of biological models. Ph.D. thesis, Shaanxi Normal University (2010)

27. Smoller, J.: Shock Waves and Reaction-Diffusion Equations. Springer, New York (1985)

28. Blat, J., Brown, K.J.: Global bifurcation of positive solutions in some systems of elliptic equations. SIAM J. Math. Anal. 17(6), 1339-1353 (1986)

29. Hess, P., Lazer, A.C.: On an abstract competition in an unstirred chemostat. Can. Appl. Math. Q. 2, 461-484 (1994)

30. Thieme, H.R.: Persistence under relaxed point-dissipativity (with application to an endemic model). SIAM J. Math. Anal. 24(2), 407-435 (1993) 